\title{
A Técnica de Geração de Colunas e Aplicações
}

\author{
Wellington Donizeti Previero
}

Orientador: Prof. Dr. Marcos Nereu Arenales

Dissertação apresentada ao Instituto de Ciências Matemáticas e de Computação - ICMC-USP, como parte dos requisitos para obtenção do título de Mestre em Ciências de Computação e Matemática Computacional.

\author{
USP - São Carlos \\ Maio/2001
}




\section{A Comissão Julgadora:}

Prof. Dr. Marcos Nereu Arenales

Prof. Dr. Reinaldo Morabito Neto

Prof. Dr. Nelson Maculan Filho

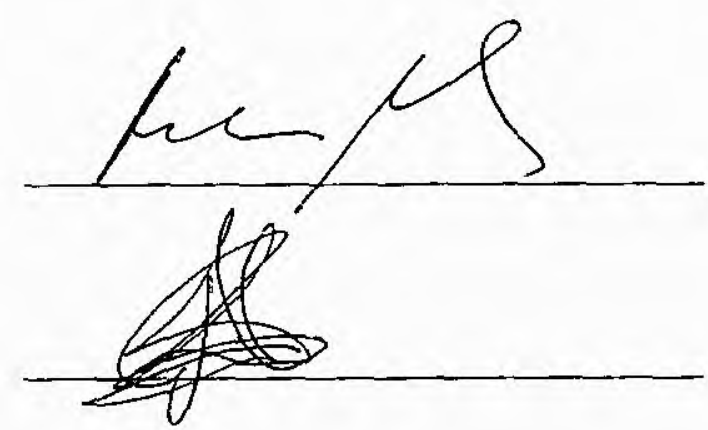

k2ken 5 
Aos meus queridos pais

José Carlos Previero e Maria Aparecida Deangele Previero, pela confiança, pelo apoio incondicional e acima de tudo, por todo o amor e carinho. 


\section{Agradecimentos}

- A Deus por está ao meu lado nos momentos de dificuldade e alegria.

- Ao professor Marcos Nereu Arenales pela amizade, confiança, incentivo permanente e por suas valiosas e inspiradoras orientações que me conduziram à realização deste trabalho. Deixo aqui registrado minha admiração!

- A FAPESP, Fundação de Ampara à Pesquisa do Estado de São Paulo, e a CAPES, Coordenação de Aperfeiçoamento Pessoal de Nível Superio, pela viabilidade financeira da pesquisa;

- Ao meu irmão Leandro e ao grande amigo Ricardo Róvero, que me apoiaram a todo momento.

- Ao meus colegas de trabalho: Thiago, Ricardo, Silvinho, Pio, Werley, Juliano, Kátia, Bibi, Rúbia, Luiz Fernando, Araxá, Maristela, Gisele, Lilian, Gláucia, Kelly, Adriano, Cecília, Kattiana e Jelder.

- Ao meus amigos de Barra Bonita: Benites, Douglas, Jean, Leonardo, Herbert, Camilo, André, Edinho e Tiago.

- A todos os professores que contribuíram diretamente para a minha formação profissional e pessoal.

- Aos funcionários do ICMC-USP pelo convívio diário, amizade e respeito.

- E finalmente, a minha Família! 


\section{Abstract}

The Technique of Column Generation is a procedure for solving large-scale optimization problems, where the number of variables are very high. This technique has advantage of working with the columns implicitly. Additional column will be generated, as needed, solving the princing problem. The Technique of Column Generation can also be used in combination with method Branch and Bound for solve problems of Integer Optimization. In this work will be presented applications of Technique of Column Generation in several problems of optimization and some computational tests. 


\section{Resumo}

A Técnica de Geração de Colunas é um procedimento para resolver problemas de otimização linear de grande escala, onde o número de variáveis é muito grande. Esta técnica tem a vantagem de trabalhar com as colunas implicitamente. Colunas adicionais serão geradas, quando necessário, resolvendo um subproblema. A Técnica de Geração de Colunas também pode ser usada em combinação com o método Branch and Bound para resolver problemas de Otimização Inteira. Neste trabalho serão apresentadas as aplicações da Técnica de Geração de Colunas em vários problemas de otimização e alguns testes computacionais. 


\section{Sumário}

$\begin{array}{ll}\text { Introdução } & 1\end{array}$

1 Revisāo Bibliográfica

2 A Técnica de Geração de Colunas 99

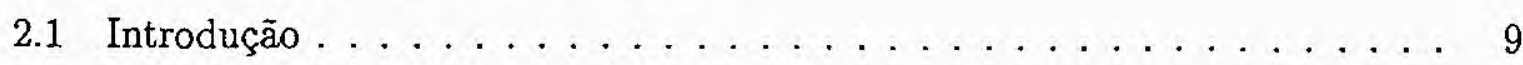

2.2 O Método Simplex com Geração de Colunas . . . . . . . . . . . . 9

2.3 O Problema de Corte de Estoque Unidimensional . . . . . . . . . . 10

2.3 .1 O Subproblema: problema da mochila inteiro . . . . . . . 12

2.4 O Problema de Designação Generalizada . . . . . . . . . . . . . . . 13

2.4 .1 O Subproblema: múltiplos problemas da mochila $0-1 \ldots \ldots$

2.5 Um problema de Roteamento de Veículos . . . . . . . . . . . . 15

2.5.1 O Subproblema: problema do caixeiro viajante e o problema da mochila . . . . . . . . . . . . . . . . 17

3 Geração de Colunas em Problemas de Otimização Inteira 19

3.1 Introdução . . . . . . . . . . . . . . . . . . . . . 19

3.2 Modelos Apropriados para a Geração de Colunas . . . . . . . . . . . 19

3.2.1 Problema Geral de Otimização Inteira . . . . . . . . . . . . . 19

3.2.2 Problemas de Particionamento de Conjuntos . . . . . . . . . 23

3.3 O Problema Reformulado . . . . . . . . . . . . . . . 25

3.3.1 Problema Geral Versus Problema Reformulado . . . . . . . . . 26

3.3.2 Relaxaçāo Lagrangiana . . . . . . . . . . . . . . . . 28

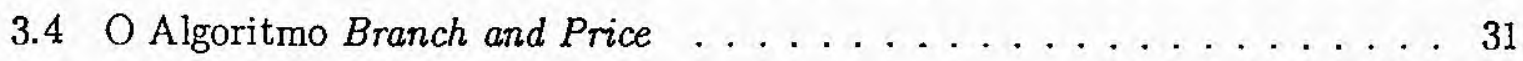

3.5 Estratégias de Ramificação . . . . . . . . . . . . . . . . . 32

3.5.1 Problema de Partição de Conjunto . . . . . . . . . . 33 
3.5.2 Problema Geral Reformulado . . . . . . . . . . . 35

3.5 .3 Problema de Designação Generalizada . . . . . . . . . . . 37

4 O Problema de Roteamento com Janela de Tempo 45

4.1 Introdução . . . . . . . . . . . . . . . . . 45

4.2 Formulação Matemática . . . . . . . . . . . . . . 46

4.3 Formulação por Partição de Conjunto . . . . . . . . . . . . . . 47

4.4 Resolução do Subproblema . . . . . . . . . . . . . . . 50

4.5 Algoritmo do Caminho Mínimo com Janela de Tempo . . . . . . . . . . 51

4.6 Resolução da Formulação por Partição de Conjuntos . . . . . . . . . . 53

5 Experiências Computacionais $\quad 55$

5.1 Introdução . . . . . . . . . . . . . . . 55

5.2 Descrição do Teste . . . . . . . . . . . . . . 55

6 Conclusões e Propostas para o Futuro $\quad 58$

$\begin{array}{ll}\text { A O Método Simplex } & 60\end{array}$

A.1 Revisão do Método Simplex . . . . . . . . . . . . . . 60

A.2 Algoritmo Primal Simplex . . . . . . . . . . . . 63

B Decomposição de Dantzig e Wolfe $\quad 64$

$\begin{array}{lll}\text { C Algoritmo de Ford-Bellman } & 68\end{array}$

$\begin{array}{ll}\text { Referências Bibliográficas } & 70\end{array}$ 


\section{Introdução}

Problemas de Otimização Combinatória e Inteira consistem em maximizar ou minimizar uma função de várias variáveis, sujeita a um conjunto de equações ou inequações e à restrição de integralidade de algumas ou todas as variáveis.

Muitos problemas de Otimização Inteira são da classe NP-completo, ou seja, não existe um algoritmo de tempo polinomial que possa resolvê-los. Portanto há grande interesse em buscar heurísticas que possam encontrar uma solução próxima da solução ótima, dentro de um intervalo de tempo razoável.

Na prática, uma abordagem comum para resolver problemas de Otimização Inteira tem sido usar o Método Simplex para resolver a relaxação do problema, e a solução obtida é arredondada para valores inteiros. A relaxação linear de um problema de Otimização Inteira é obtida pela omissão da restrição de integralidade. Durante toda a dissertação, quando citarmos o termo relaxação de um determinado problema, estamos nos referindo à relaxação linear. Embora muitas vezes seja adequada, existem falhas nesta abordagem. Uma é que a solução ótima da relaxação do problema de Otimização Inteira não é necessariamente factível após o arredondamento. Frequentemente é difícil de realizar o arredondamento e manter a factibilidade da solução. Mesmo que a solução da relaxação do problema de Otimização Linear seja arredondada com sucesso, não teremos a garantia que está solução seja uma soluçāo ótima para problema de Otimização Inteira. $\mathrm{Na}$ verdade ela pode estar longe do ótimo, do ponto de vista da função objetivo.

$\mathrm{Na}$ última década, o uso de problemas de Otimização Inteira tem mudado e aumentado bruscamente. A possibilidade de resolver problemas relativamente grandes em pequena quantidade de tempo, vem tornando a Otimização Inteira muito mais comum. Quinze anos atrás, os computadores eram usados para resolver problemas com centenas de variáveis inteiras. Agora é possível resolver problemas com milhares de variáveis inteiras num computador pessoal ou numa estação de trabalho. Podemos ainda conseguir, 
heuristicamente, boas soluções aproximadas para o problema. Estas vantagens têm sido possíveis devido ao desenvolvimento da computação e a algoritmos especializados.

Existem problemas de Otimização Inteira que apresentam características que inviabilizam a utilização do Método Simplex em sua resolução: o número de variáveis é extremamente grande. A técnica de geração de colunas vem suprir essa dificuldade, pois tem a vantagem de trabalhar com as colunas implicitamente.

O pioneiros a utilizarem a técnica de geração de colunas foram Dantzig e Wolfe (1960) e Gilmore e Gomory $(1961,1963)$. O trabalho de Dantzig e Wolfe (1960) ficou conhecido como o Princípio da Decomposição de Dantzig e Wolfe e consiste num procedimento para resolver problemas de Otimização Linear de grande escala, que contém restrições com estruturas especiais. Gilmore e Gomory $(1961,1963)$ utilizaram o conceito de padrão de corte para resolver o problema de corte de estoque unidimensional. O problema original de corte de estoque era um problema de Otimização Inteira e a técnica de geração de colunas aplicada à sua relaxação forneceu, na prática, um ótimo limitante para o problema original.

Recentemente a técnica de geração de colunas tem sido usada para resolver problemas de Otimização Inteira. A solução inteira é obtida combinando o método de enumeração implícita (branch and bound) e a geração de colunas para resolver a relaxação do problema de Otimização Inteira em cada nó da árvore. Este procedimento recebeu o nome da língua inglesa de Branch and Price.

Um importante acontecimento para o desenvolvimento do estudo do algoritmo Branch and Price foi a publicação do trabalho de Barnhart et al. (1998). Nesse trabalho, os autores apresentaram uma estrutura geral, unificando vários trabalhos usando tal técnica. Os autores desse trabalho são atualmente os que mais têm publicado sobre o método Branch and Price e, com certeza, o mesmo é um marco na área de Programa Inteira. Devido à sua importância, o trabalho de Barnhart et al. (1998) foi bastante detalhado nesta dissertação.

Neste trabalho apresentaremos com detalhes a utilização da técnica de geração de colunas em várias classes de problemas, bem como a sua utilização e dificuldade para resolver Problemas de Otimização Inteira. As experiências computacionais tomam como base o modelo proposto por Desrosiers et al. (1984) para o Problema de Roteamento com Janela de Tempo. 
A dissertação está organizada na seguinte maneira:

- Capítulo 1: é feita uma revisão bibliográfia da aplicação técnica de geração de colunas;

- Capítulo 2: é apresentado como a técnica de geração de colunas é utilizada com Método Simplex. Três modelos serão utilizados para exemplificar o subproblema de geração de colunas;

- Capítulo 3: está detalhada a utilização da técnica de geração de colunas em Problemas de Otimização Inteira;

- Capítulo 4: é definido e modelado o Problema de Roteamento com Janela de Tempo, o qual será utilizado para as experiências computacionais;

- Capítulo 5: experiências computacionais são apresentadas;

- Capítulo 6: a dissertação é concluída e apresentadas algumas perspectivas futuras. 


\section{Capítulo 1}

\section{Revisão Bibliográfica}

Neste capítulo revisamos a utilização da técnica de geração de colunas em alguns problemas encontrados na literatura.

A técnica de geração de colunas foi utilizada no início do anos $60 \mathrm{em}$ problemas de otimização de grande porte com estruturas especiais. Os pioneiros a utilizarem essa técnica foram Dantzig e Wolfe (1960), cujo método recebeu o nome de Princípio da Decomposição de Dantzig e Wolfe. Nesse trabalho, o problema de Otimização Linear com um número grande de restrições é transformado em outro com um número grande de variáveis. Gilmore e Gomory (1961), (1963) utilizaram a técnica para resolver a relaxação do problema de corte de estoque. O problema original de corte de estoque era um problema de Otimização Inteira e a técnica de geração de colunas aplicada à sua relaxação forneceu, na prática, um ótimo limitante para o problema original.

Devido ao fato de a técnica de geração de colunas aplicada a relaxações de problemas de Otimização Inteira produzir ótimos limitantes, a sua utilização pode ser encontrados em diversos trabalhos.

Recentemente, o método de geração de colunas tem sido usado para resolver otimamente problemas de Otimização Inteira. A solução inteira é obtida combinando o método de enumeração implícita (branch and bound) e a geração de colunas para resolver a relaxação do problema de Otimização Inteira em cada nó da árvore. A geração de colunas, como veremos, corresponde à resolução de um subproblema que absorve parte da natureza combinatória do problema original. Em cada nó da árvore devem ser elaboradas estratégias de ramificação (onde novas restriçōes são adicionadas) para que a estrutura do subproblema seja mantida. Esta técnica de resolução recebeu o nome na literatura de 
língua inglesa de Branch and Price.

Um trabalho que merece destaque, sobre geração de colunas em Otimização Inteira, encontra-se em Barnhart et al. (1998). Nesse trabalho, os autores procuraram unificar os vários trabalhos da literatura, apresentando uma estrutura geral para a geração de colunas e dando idéias gerais sobre a sua utilização em vários contextos.

Em Brito et al. (2000) é apresentado um método de geração de colunas para problemas de Otimização Linear Inteira, o qual usa o algoritmo primal e dual simplex. Nesse trabalho é apresentado um levantamento bibliográfico da técnica de geração de colunas e que será utilizado neste capítulo. Outros trabalhos não relacionados em Brito et al. (2000) são incluídos.

A técnica de geração de colunas pode ser observada com grande sucesso em problemas de roteamento de veículos, bem como nos trabalhos de Desrosiers et al. (1984), Desrochers et al. (1992), Ribeiro e Soumis (1994), Desrosiers et al. (1995), Bramel e Levi (1997), Lobel (1998) e Desrosiers et al.(1999). Desrosiers et al. (1984) foram uns dos primeiros na literatura a utilizar o método de enumeração implícita com geração de colunas. Nesse trabalho, um problema de roteamento é formulado como um problema de partição de conjuntos e as colunas são geradas resolvendo o problema do caminho mínimo com janela de tempo. Ribeiro e Soumis (1994) utilizaram o método de geração de colunas para resolver a relaxação do problema de programação de veículos com múltiplos depósitos, obtendo um limitante melhor ao que se tinha conhecido na literatura.

Desrosiers et al. (1995) concluíram que algoritmos relacionados com a decomposição Dantzig e Wolfe e técnicas de geração de colunas têm demonstrado ser os mais eficientes para obter soluções para problemas de roteamento com restrições temporais.

Vários trabalhos na área de problemas de corte de estoque podem ser encontrados na literatura utilizando a geração de colunas. Barnhart al. (1994) utilizaram o algoritmo Branch and Price para obter uma solução inteira para o problema binário de corte de estoque unidimensional. Eles propuseram uma regra de ramificação que mantém a estrutura do problema em cada nó da árvore. Em Vance (1998) foram comparados dois algoritmos para o problema de corte de estoque unidimensional. Cada algoritmo é baseado em duas formulações diferentes do problema mestre. Ambos os algoritmos utilizam o Branch and Price, o qual produz o mesmo subproblema, mas necessitam de diferentes abordagens de ramificação. Carvalho (1998) apresentou uma abordagem diferente para o 
problema geral de corte de estoque. O problema de corte de estoque é formulado como fluxo em redes. Vanderbeck (1999) apresentou um estudo computacional do algoritmo de geração de colunas para o problema de corte de estoque e bin packing.

Mehrotra e Trick (1996) desenvolveram um método para resolver o problema de coloração de grafos usando a formulação de conjuntos in dependentes. Mehrotra e Trick (1998) também utilizaram a técnica de geração de colunas para o problema de agrupamento restrito com uma estratégia especial de ramificação, e os resultados obtidos mostraram uma significante melhora em exemplares difíceis do problema.

Savelsbergh (1997) utilizou o algoritmo Branch and Price para obter a solução ótima inteira para o problema de designação generalizada. Em seu trabalho, o problema de designação generalizada é formulado como um problema de partição de conjunto.

Vanderbeck e Wolsey (1996) apresentaram estratégias especiais de ramificação parạ problema gerais. Em Vanderbeck (2000) é realizado um estudo da decomposição Dantzig e Wolfe em Problemas de Otimização Inteira. Também são propostas novas estratégias de ramificação e citado o efeito na estrutura do subproblema.

Em Barnhart et al. (2000), o problema de multifluxo é resolvido combinando a geração de colunas, regras de ramificação e planos de cortes. A combinação dessas técnicas é mutuamente compatível ao longo da árvore do branch and bound e, até o momento, poucos trabalhos podem ser encontrados na literatura que utilizem esta combinação.

Em Van Den Akker et al. (2000), a geração de colunas é utilizada para problemas de sequenciamento de máquinas com formulações indexadas no tempo. Os autores mostraram que a Decomposição Dantzig e Wolfe pode ser usada com eficiência para diminuir as dificuldades associadas ao tamanho do problema. Além disso, mostraram que é possível, usando a Decomposição Dantzig e Wolfe, calcular uma solução boa para a relaxação do problema com grande rapidez. Em Cattrysse et al. (1993), o problema de sequenciamento e dimensionamento de lotes discreto é formulado com partição de conjuntos, empregando a técnica de geração de colunas em sua resolução. Os resultados computacionais obtidos mostraram que os métodos são muitos eficientes, tanto na qualidade das soluções como na exigência da memória e tempo computacional.

E, por fim, em Desrosiers et al. (1999) é proposto um algoritmo para melhorar a convergência do processo de solução ao se utilizar a geração de colunas, baseado no algoritmo de Kelley. Resultados preliminares obtidos em problemas de localização e transporte 
aéreo mostraram que o algoritmo pode ser usado para melhorar o tempo de resolução de exemplares difíceis e para resolver alguns problemas grandes. 


\section{Capítulo 2}

\section{A Técnica de Geração de Colunas}

\subsection{Introdução}

Neste capítulo apresentaremos como a técnica de geração de colunas pode ser utilizada com o Método Simplex em problemas de grande porte. Cada coluna gerada é solução de um subproblema. A característica do subproblema depende do problema; a seguir ilustraremos três modelos com os seus respectivos subproblemas. Os modelos apresentados são: O Problema de Corte de Estoque Unidimensional, O Problema de Designação Generalizada e um Problema de Roteamento. Para esses modelos nāo enfocaremos a técnica de resolução, mas os seus subproblemas. O Problema de Designação Generalizada também será utilizado no Capítulo 3 para exemplificar as dificuldades encontradas nas estratégias de ramificação.

\subsection{Método Simplex com Geração de olunas}

Considere o problema de otimização linear sendo que o número de variáveis $n$ é muito maior que o número de restrições $m$. A enumeração completa de todas as colunas é, para muitos problemas, um trabalho computacionalmente impossível, pois pode atingir vários milhões ou bilhões de colunas. Entretanto, apenas $m$ colunas na matriz $A$ são necessárias para descrever uma solução ótima, isto é, uma solução básica.

Ao aplicarmos o Método Primal Simplex (para maiores detalhes do Método Primal Simplex, veja o Apêndice $A$ ), necessitamos de uma base primal factível $B$ que produza a solução básica factível 


$$
x=\left(x_{B}, x_{N}\right)
$$

com $x_{B}=B^{-1} b$ e $x_{N}=0$, e o vetor multiplicador simplex

$$
\pi^{T}=c_{B} B^{-1}
$$

Para verificar a otimalidade da solução básica $x$, determinamos a variável $x_{k}$ com o menor custo relativo, o que sugere o seguinte subproblema:

$$
c_{k}-\pi^{T} a_{k}=\text { Mínimo }\left\{c_{j}-\pi^{T} a_{j}, j=1, \ldots, n\right\}
$$

Considere $a=\left(\alpha_{1}, \alpha_{2}, \ldots, \alpha_{m}\right)$ uma coluna da matriz $A$ e suponha que o coeficiente da função objetivo seja dado por:

$$
c(a)=f(a)+\sum_{i=1}^{m} \gamma_{i} \alpha_{i}
$$

onde $\gamma_{i} \in R, i=1, \ldots, m$ são conhecidos e $f(a)$ é uma variável em função dos elementos da coluna $a$. Assim o custo relativo da variável, cuja coluna é dada por $a$, pode ser determinado por:

$$
c(a)-\pi^{T} a=f(a)+\sum_{i=1}^{m} \gamma_{i} \alpha_{i}-\sum_{i=m}^{m} \pi_{i} \alpha_{i}=f(a)+\sum_{i=1}^{m}\left(\gamma_{i}-\pi_{i}\right) \alpha_{i}
$$

e o subproblema de determinar a coluna com o menor custo relativo pode ser reescrito por:

$$
\begin{array}{ll}
\text { Minimizar } & f(a)+\sum_{i=1}^{m}\left(\gamma_{i}-\pi_{i}\right) \alpha_{i} \\
\text { Sujeito a } & \left(\alpha_{1}, \alpha_{2}, \ldots, \alpha_{m}\right) \in X
\end{array}
$$

onde $X \in N^{m}$ é o conjunto que descreve as propriedades da coluna $a \in A$.

\subsection{O Problema de Corte de Estoque Unidimensional}

O Problema de Corte de Estoque consiste em atender a um pedido de produção de itens a partir da cortagem de objetos em estoque otimizando uma função objetivo, e por exemplo, minimizando as perdas. Gilmore e Gomory (1961) desenvolveram a clássica 
abordagem do problema por Otimização Linear Inteira. Em seus estudos eles utilizaram o conceito de padrões de corte e resolveram a relaxação do problema por geração de colunas. Heurísticas são usadas para obter a solução inteira, a qual não é necessariamente a solução ótima. Wäscher e Gau (1996) realizaram um estudo computacional sobre tais heurísticas para o problema unidimensional. O Problema de Corte é considerado unidimensional quando apenas uma dimensão é relevante no processo de cortagem.

Para formular o problema, consideramos objetos de comprimento $L$ com quantidade suficiente para atender toda a demanda. A produção consiste em fabricar um determinado número $N_{i}$ de itens de comprimento $l_{i}$ do material estocado, com $i=1, \ldots, m$. Um custo $c$ é atribuído a cada objeto estocado e o custo de atender a demanda é simplesmente a soma dos custos do objetos cortados

Definição 1: Chamamos de padrão de corte a maneira como um objeto em estoque é cortado para a produção de itens demandados. A um padrão de corte associamos um vetor $m$-dimensional que contabiliza os itens produzidos:

$$
a=\left(a_{1}, a_{2}, \ldots, a_{m}\right)
$$

onde $a_{i}$ é um inteiro não-negativo que indica a quantidade de itens do tipo $i$ segundo o padrão de corte.

Para definir todos os padrões de corte é necessário determinar todas as possíveis soluções de

$$
\begin{aligned}
& l_{1} a_{1}+l_{2} a_{2}+\ldots l_{m} a_{m} \leq L \\
& a_{i} \geq 0 \text { e inteiro } \quad i=1, \cdots, m .
\end{aligned}
$$

Suponha que existam $n$ possíveis soluções do sistema (2.2), dados por :

$$
a_{1}=\left(\begin{array}{c}
a_{11} \\
a_{21} \\
\cdot \\
\cdot \\
\cdot \\
a_{m 1}
\end{array}\right) \quad a_{2}=\left(\begin{array}{c}
a_{12} \\
a_{22} \\
\cdot \\
\cdot \\
\cdot \\
a_{m 2}
\end{array}\right) \quad \ldots a_{n}=\left(\begin{array}{c}
a_{1 n} \\
a_{2 n} \\
\cdot \\
\cdot \\
\cdot \\
a_{m n}
\end{array}\right) .
$$


Seja $x_{j}$ o número de objetos em estoque cortados segundo o padrão $j$. Temos então o problema de Otimização Linear:

$$
\begin{array}{ll}
\text { Minimizar } & \sum_{j=1}^{n} c x_{j} \\
\text { Sujeito } a & \sum_{j=1}^{n} a_{j} x_{j}=N \\
& x_{j} \geq 0 \text { e inteiro, } \quad j=1, \cdots, n .
\end{array}
$$

Observe que minimizar a função objetivo custo, neste caso, corresponde a minimizar o número de objetos cortados (Minimizar $f(x)=x_{1}+x_{2}+\ldots+x_{n}$ ).

Há dois fatores que tornam a resolução do problema de corte de estoque inviável. Primeiro a restriçāo de integralidade. Segundo, em problemas práticos o número de variáveis pode ser da ordem de centenas de milhares. A exigência de integralidade sobre as variáveis $x_{j}$ torna o problema de atender a demanda difícil, senão impossível de ser resolvido computacionalmente. Entretanto, o truncamento das soluções não inteiras (infactíveis) "resolve", de certa forma, boa parte do problema, restando poucos itens para serem cortados, sendo pouco significativos do ponto de vista da perda total ou custo. Em relação ao problema de número de variáveis, a técnica de geração de colunas pode superar essa dificuldade.

\subsubsection{O Subproblema: problema da mochila inteiro}

Consideremos o problema (2.3), onde as barras de comprimento $L$ devern ser cortadas em pedaços $l_{i}, i=1, \ldots, \mathrm{m}$. As colunas da matriz $A$ são bern determinadas pelo conjunto

$$
X=\left\{a \in R^{m} \mid l_{1} a_{1}+l_{2} a_{2}+\cdots+l_{m} a_{m} \leq L, a_{i} \geq 0 \text { e inteiro, } i=1, \ldots, m\right\}
$$

Considere uma base formada por colunas de $A$, digamos $B$ ( $B$ é formada por $m$ colunas liniarmente independentes). O vetor multiplicador simplex é facilmente calculado por $\pi^{T}=c_{B}^{T} B^{-1}$ (note que $c_{B}^{T}=(1, \ldots, 1)$, pois os custo de qualquer variável é igual a 1 , considerando $\left.f(x)=x_{1}+x_{2}+\ldots+x_{n}\right)$.

O subproblema (2.1), de determinar o menor custo relativo é dado por: 


$$
\begin{array}{ll}
\text { Minimizar } & 1-\sum_{i=1}^{m} \pi_{i} \alpha_{i} \\
\text { Sujeito } a & \sum_{i=1}^{m} l_{i} \alpha_{i} \leq L \\
& \alpha_{i} \geq 0 \text { e inteiro } \quad i=1, \cdots, m
\end{array}
$$

ou, equivalentemente,

$$
\begin{array}{ll}
\text { Maximizar } & \sum_{i=1}^{m} \pi_{i} \alpha_{i} \\
\text { Sujeito a } & \sum_{i=1}^{m} l_{i} \alpha_{i} \leq L \\
& \alpha_{i} \geq 0 \text { e inteiro } \quad i=1, \cdots, m,
\end{array}
$$

o qual é o clássico Problema da Mochila Inteiro.

Portanto, resolvendo o problema de Corte de Estoque Unidimensional pela formulação (2.3), a cada iteração do Método Simplex será necessário resolver o Problema da Mochila Inteiro para determinar a coluna com o menor custo relativo.

\subsection{O Problema de Designação Generalizada}

O Problema de Designação Generalizada consiste em determinar o lucro máximo ao se designar $n$ tarefas para $m$ máquinas, tal que cada tarefa seja atribuída precisamente a uma máquina e cada máquina esteja limitada pela sua capacidade. A formulação padrão do problema de Otimização Inteira é a seguinte:

$$
\begin{array}{ll}
\text { Maximizar } & \sum_{i=1}^{m} \sum_{j=1}^{n} p_{i j} z_{i j} \\
\text { Sujeito a } & \sum_{j=1}^{n} z_{i j}=1, \quad i=1, \ldots, m \\
& \sum_{i=1}^{m} w_{i j} z_{i j} \leq d_{j}, \quad j=1, \ldots, n \\
& z_{i j} \in\{0,1\}, \quad i=1, \ldots, m, \quad j=, 1 \ldots, n .
\end{array}
$$

onde $p_{i j}>0$ é o desempenho da máquina $i$ em realizar a tarefa $j, w_{i j}>0$ é a quantidade de capacidade da máquina ao se realizar a tarefa $j$ na máquina $i, d_{j}>0$ é capacidade da 
máquina $j$ e $z_{i j}$ a variável binária que indica se a tarefa $i$ é designada para a máquina $j$ $\left(z_{i j}=1\right)$ ou não $\left(z_{i j}=0\right)$

A matriz de restrições do problema (2.6) possui a estrutura bloco-angular, a qual é explorada na Decomposição Dantzig e Wolfe.

Seja $K^{j}=\left\{y_{1}^{j}, y_{2}^{j}, \ldots, y_{K_{j}}^{j}\right\}$ o conjunto de todas as possíveis designações de tarefas para a máquina $j$, onde $y_{k}^{j}=\left(y_{1 k}^{j}, y_{2 k}^{j}, \ldots, y_{m k}^{j}\right)$ satisfaz a restrição:

$$
\begin{aligned}
& \sum_{i=1}^{n} w_{i j} y_{i k}^{j} \leq d_{j} \\
& y_{i k}^{j} \in\{0,1\}, \quad i=1, \ldots, m .
\end{aligned}
$$

Temos que $y^{j} \in K^{j}$ se, e somente se:

$$
\begin{aligned}
& y^{j}=\sum_{k=1}^{K_{j}} y_{k}^{j} \lambda_{k}^{j}, \\
& \sum_{k=1}^{K_{j}} \lambda_{k}^{j}=1, \\
& \lambda_{k}^{j} \in\{0,1\} .
\end{aligned}
$$

Substituindo as restrições (2.7) no Problema Original (2.6) temos o Problema Reformulado

$$
\begin{array}{ll}
\text { Maximizar } \quad & \sum_{j=1}^{n} \sum_{k=1}^{K_{j}}\left(\sum_{i=1}^{m} p_{i j} y_{i k}^{j}\right) \lambda_{k}^{j} \\
\text { Sujeito a } \quad & \sum_{j=1}^{n} \sum_{k=1}^{K_{j}} y_{i k}^{j} \lambda_{k}^{j}=1 \quad i=1, \ldots, m \\
& \sum_{k=1}^{K_{j}} \lambda_{k}^{j}=1 \quad j=1, \ldots, n \\
& \lambda_{k}^{j} \in\{0,1\} \quad i=1, \ldots, m, \quad j=1, \ldots, n .
\end{array}
$$

\subsubsection{O Subproblema: múltiplos problemas da mochila 0-1}

Supondo que seja conhecida uma base $B$ para a relaxação do Problema Reformulado (2.8), o Problema de Designação Generalizada pode ser resolvido utilizando o Método 
Simplex. O vetor multiplicador simplex será denotado por $(\mu, \nu)$, onde $\mu \in R^{m}$ e $\nu \in R^{n}$. Como o problema é maximizar a função objetivo, a coluna que entrará na base será a de maior custo relativo. Neste caso, a coluna com o maior custo relativo será determinada pelo seguinte subproblema

$$
\text { Maximizar }_{1 \leq j \leq n}\left\{z\left(M O C H_{j}\right)-\nu_{j}\right\}
$$

onde $\nu_{j}$ é o vetor multiplicador simplex do Problema Reformulado Restrito associado à restrição de convexidade da maquina $j$ e $z\left(M O C H_{j}\right)$ é a solução ótima do seguinte Problema da Mochila 0-1:

$$
\begin{array}{lll}
z\left(\mathrm{MOCH}_{j}\right)=\text { Máximo } & \sum_{i=1}^{m}\left(p_{i j}-\mu_{i}\right) y_{i}^{j} \\
\text { Sujeito a } & \sum_{\substack{i=1 \\
m}}^{m} w_{i j} y_{i}^{j} \leq d_{j} \\
& y_{i}^{j} \in\{0,1\}, \quad i=1, \ldots, m .
\end{array}
$$

Qualquer coluna com o maior custo reduzido é candidata a entrar na base. Entretanto, resolver o subproblema envolve a solução de vários Problemas da Mochila 0-1, o que pode ser computacionalmente inviável. Além disso, não precisamos escolher necessariamente a coluna com o maior custo relativo, qualquer coluna com custo relativo positivo pode ser tomada para melhorar a solução.

Segundo Barnhart et al. (1998), várias alternativas de selecionar a coluna com custo relativo positivo podem ser escolhidas. Uma alternativa b́bvia consiste em selecionar a primeira coluna encontrada com custo relativo positivo. Este procedimento, reduz o tempo computacional por iteração de Método Simplex. Entretanto, desde que o número de iteraçōes pode aumentar, este procedimento pode ser ruim. Outra alternativa é selecionar todas as colunas encontradas com custo relativo positivo. A cada iteração do Método Simplex esses conjuntos de colunas são adicionados à matriz do sistema.

\subsection{Um problema de Roteamento de Veículos}

Sierksma e Tijssen (1998) apresentaram um modelo cujo propósito foi desenvolver um algoritmo para atender a demanda de trocas de funcionários em várias plataformas 
marítimas, partindo e retornando à mesma base terrestre, de tal modo que o custo total de transporte seja o menor possível.

Para a formulação matemática do modelo, será utilizada a seguinte notação:

$N=$ número de plataforma;

$N_{f}=$ número de vôo factível;

$i=$ índices da plataforma, $i=1, \ldots, N$;

$f=$ índice do vôo, $f=1, \ldots, N_{f}$;

$x_{f}=$ número de vezes que o vôo $f$ é realizado;

$D_{f}=$ número de trocas de equipes na plataforma;

$a_{i f}=$ número de trocas de equipes na plataforma $i$ durante o vôo $f$;

$d_{f}=$ custo de realizar um vôo;

$C=$ capacidade do helicóptero.

É suposto que o custo de realizar um vôo seja uma função linear do total da distância percorrida. Ainda mais, é utilizada a distância total percorrida durante o vôo. A distância total percorrida $d_{f}$ durante o vôo $f$ é definida como o comprimento da rota, que inclui o aeroporto e todas as plataformas visitadas (Problema do Caixeiro Viajante).

Umn vôo factível é aquele cujo raio de alcance $R$ não é ultrapassado e o número de trocas durante o vôo não exceda a capacidade $C$ do helicóptero. Dois vôos factíveis visitando o mesmo conjunto de plataformas, mas com diferentes trocas, são considerados como vôos factíveis diferentes.

Claramente, para um conjunto de plataforma e aeroporto, existem várias possibilidades de vôos factíveis, sempre com a possibilidade de determinar a menor rota possível, ou seja, um Problema do Caixeiro Viajante devendo ser resolvido.

O número $N_{f}$ de todos os vôos possíveis cresce exponencialmente de acordo com o número de plataformas e a capacidade do helicóptero. O problema pode ser formulado como um modelo de Otimização Linear Inteira:

$$
\begin{array}{ll}
\text { Minimizar } & \sum_{f=1}^{N_{f}} d_{f} x_{f} \\
\text { Sujeito a } & \sum_{f=1}^{N_{f}} a_{i f} x_{f}=D_{i}, \quad i=1, \ldots, N \\
& x \geq 0 \text { e inteiro, } \quad f=1, \ldots, N_{f} .
\end{array}
$$


A função objetivo é o somatório de $d_{f} x_{f}$ sobre todos os vôos factíveis $f$, onde $d_{f}$ é comprimento do vôo $f=1, \ldots, N_{f}$. O número total de passageiros com destino à plataforma $i$ em todos os helicópteros tem de ser igual a $D_{i}$. A matriz $A=\left\{a_{i j}\right\}$ do modelo tem $N$ linhas e $N_{f}$ colunas. Uma partição básica inicial pode ser construída tomando a cada vôo um único passageiro na plataforma $i, i=1, \ldots, N$. Desta forma a temos como partição básica inicial a matriz identidade. Como $N_{f}$, o número de variáveis, é muito grande, o procedimento de geração de colunas pode ser bem adequado para resolver o problema relaxado.

\subsubsection{O Subproblema: problema do caixeiro viajante e o pro- blema da mochila}

Considere uma partição $A=(B, N)$ do problema $(2.10)$ e o vetor multiplicador simplex dado por $\pi=d_{f_{B}} B^{-1}$, onde $d_{f_{B}}$ é o vetor dos cumprimentos dos vôos básicos. O custo relativo da f-ésima coluna é dado por

$$
d_{f}-\sum_{i=1}^{N} \pi_{i} a_{i f}
$$

De acordo com (2.1), para determinar a coluna com o menor custo relativo, temos que resolver o seguinte subproblema:

$$
\begin{array}{ll}
\text { Minimizar } & d_{f}-\sum_{i=1}^{N} \pi_{i} a_{i} \\
\text { Sujeito } a & \sum_{i=1}^{N} a_{i} \leq C \\
& 0 \leq a_{i} \leq D_{i}, \quad i=1, \ldots, N \\
& d_{f} \leq R
\end{array}
$$

As variáveis de decisão do modelo acima (2.11) são $a_{i}, i=1, \ldots, N$ e $d_{f}$. Como $d_{f}$, a distância percorrida pelo vôo $f$, depende dos valores não nulos de $a_{i}$, o subproblema (2.11) é um modelo não linear de dificil resoluçāo.

Definindo $S=\left\{P_{i} \mid a_{i}>0\right\}$ como o conjunto das plataformas com trocas positivas, então $d_{f}$ é a menor distância entre o aeroporto a todas as plataformas de $S$.

Logo, Dara resolver o subproblema (2.11), são realizados os seguintes passos: 
Passo 1: Fixe um subconjunto de plataformas S. Resolva o Problema do Caixeiro Viajante sobre o subconjunto de $S$ para determinar o valor $d_{f}(S)$ (elimine todos os subconjuntos de plataformas dominados por $S$, veja Sierksma e Tijssen (1998)). Obviamente, o número de subconjuntos $S$ pode ser muito grande e heurísticas devem ser desenvolvidas (Sierksma e Tijssen, (1998)). Como $d_{f}(S)$ tornou-se uma constante, temos:

se $d_{f(s)}>R$, então o conjunto $S$ não possui um vôo factível.

se $d_{f(S)} \leq R$, então:

Passo 2: A função objetivo do subproblema (2.11) tem a seguinte forma:

$$
d_{f(S)}-\sum_{i \in S} \pi_{i} a_{i}
$$

Determinados os passos acima, resolver o subproblema (2.11) equivale a resolver o Problema da Mochila

$$
\begin{array}{ll}
\text { Maximizar } & \sum_{i \in S} \pi_{i} a_{i} \\
\text { Sujeito a } & \sum_{i \in S}^{N} a_{i} \leq C, \\
& 0 \leq a_{i} \leq D_{i}, \quad i \in S .
\end{array}
$$

Então, para este modelo de Roteamento de Veículos, o subproblema consiste em resolver, primeiramente, o Problema do Caixeiro Viajante e depois o Problema da Mochila. 


$$
\begin{array}{ll}
\text { Maximizar } & c^{T} x \\
\text { Sujeito a } & A x \leq b \\
& x \in S \\
& x \text { Inteiro }
\end{array}
$$

Consideremos que o número de linhas da matriz $A$ seja igual a $m$. Barnhart et al. (1998) consideram que a função objetivo do problema (3.1) pode ser não linear, mas considerando tal hipótese, ao omitir a restrição $A x \leq b$, a relaxação do problema deve ser de fácil resolução.

A idéia fundamental da geração de colunas é que o conjunto $S^{*}=\{x \in S: x$ inteiro $\}$ pode ser representado por um conjunto finito de vetores. Por exemplo, se $S$ é limitado então $S^{*}$ satisfaz a propriedade; ou se $x$ é binário, então $S^{*}$ é o conjunto de pontos extremos do envoltório convexo, denotado por $\operatorname{conv}\left(S^{*}\right)$.

Quando $S$ é não limitado, o resultado clássico de Minkowski e Weyl (conforme Nemhauser e Wolsey (1988)) afirma que o conv $\left(S^{*}\right)$ pode ser representado pela combinação finita de um conjunto de vetores e uma combinação linear finita do conjunto de raios. Entretanto, por questão de simplicidade, admitiremos que o conjunto $S$ é limitado. Vanderbeck (1994),(1995) fornecem mais detalhes para o caso não limitado.

Para diferenciar as variáveis do problema (3.1) e da sua reformulação, consideremos $S^{*}=\left\{y_{1}, \ldots, y_{p}\right\}$, onde $S^{*}=\{x \in S: x$ inteiro $\}$. Qualquer ponto $y \in S^{*}$ pode ser representado como

$$
\begin{aligned}
& y=\sum_{k=1}^{p} y_{k} \lambda_{k}, \\
& \sum_{k=1}^{p} \lambda_{k}=1, \\
& \lambda_{k} \in\{0,1\}, \quad k=1, \ldots, p .
\end{aligned}
$$

Sejam $c_{k}=c\left(y_{k}\right)$ e $a_{k}=A y_{k}$. Substituindo as restrições (3.2) no Problema Geral (3.1), temos o Problema Reformulado ou Problema Mestre 


$$
\begin{array}{ll}
\text { Maximizar } & c^{T} x \\
\text { Sujeito a } & A x \leq b \\
& x \in S \\
& x \text { Inteiro }
\end{array}
$$

Consideremos que o número de linhas da matriz $A$ seja igual a $m$. Barnhart et al. (1998) consideram que a função objetivo do problema (3.1) pode ser não linear, mas considerando tal hipótese, ao omitir a restrição $A x \leq b$, a relaxação do problema deve ser de fácil resoluçāo.

A idéia fundamental da geração de colunas é que o conjunto $S^{*}=\{x \in S: x$ inteiro $\}$ pode ser representado por um conjunto finito de vetores. Por exemplo, se $S$ é limitado então $S^{*}$ satisfaz a propriedade; ou se $x$ é binário, então $S^{*}$ é o conjunto de pontos extremos do envoltório convexo, denotado por conv $\left(S^{*}\right)$.

Quando $S$ é não limitado, o resultado clássico de Minkowski e Weyl (conforme Nemhauser e Wolsey (1988)) afirma que o $\operatorname{conv}\left(S^{*}\right)$ pode ser representado pela combinação finita de um conjunto de vetores e uma combinação linear finita do conjunto de raios. Entretanto, por questão de simplicidade, admitiremos que o conjunto $S$ é limitado. Vanderbeck (1994),(1995) fornecem mais detalhes para o caso não limitado.

Para diferenciar as variáveis do problema (3.1) e da sua reformulação, consideremos $S^{*}=\left\{y_{1}, \ldots, y_{p}\right\}$, onde $S^{*}=\{x \in S: x$ inteiro $\}$. Qualquer ponto $y \in S^{*}$ pode ser representado como

$$
\begin{aligned}
& y=\sum_{k=1}^{p} y_{k} \lambda_{k}, \\
& \sum_{k=1}^{p} \lambda_{k}=1, \\
& \lambda_{k} \in\{0,1\}, \quad k=1, \ldots, p .
\end{aligned}
$$

Sejam $c_{k}=c\left(y_{k}\right)$ e $a_{k}=A y_{k}$. Substituindo as restrições (3.2) no Problema Geral (3.1), temos o Problema Reformulado ou Problema Mestre 


$$
\begin{array}{ll}
\text { Maximizar } & \sum_{k=1}^{p} c_{k} \lambda_{k} \\
\text { Sujeito a } & \sum_{k=1}^{p} a_{k} \lambda_{k} \leq b \\
& \sum_{k=1}^{p} \lambda_{k}=1 \\
& \lambda_{k} \in\{0,1\}, \quad k=1, \ldots, p
\end{array}
$$

Durante toda a dissertação será utilizado o termo Problema Reformulado, ao invés de Problema Mestre. O subproblema de determinar a coluna com o maior custo relativo será dado por:

$$
\begin{array}{ll}
\text { Maximizar } & c(y)-\left(\pi^{T} A\right) y-\nu \\
\text { Sujeito a } & y \in S^{*}
\end{array}
$$

onde $(\pi, \nu)$, com $\pi \in R^{m}$ e $\nu \in R$, é o vetor multiplicador simplex.

Se considerarmos a função objetivo do Problema Geral não linear, o seu Problema Reformulado é idêntico ao problema (3.3). Note que o Problema Reformulado (3.3) é um problema de Otimização Linear Inteira, enquanto o Problema Geral possui a função objetivo não linear. A linearização é possível pois na equação

$$
y=\sum_{k=1}^{p} y_{k} \lambda_{k}
$$

obteremos $y=y_{k}$ para algum $k$, uma vez que $\sum_{k=1}^{p} \lambda_{k}=1$ e $\lambda_{k} \in\{0,1\}$.

Se o conjunto $S$ pode ser decomposto, ou seja $S=\cup_{j=1}^{n} S_{j}$, podemos representar cada conjunto

$$
S_{j}^{*}=\left\{x_{j} \in S_{j}: x_{j} \text { inteiro }\right\}
$$

como

$$
S_{j}^{*}=\left\{y_{1}^{j}, \ldots, y_{p_{j}}^{j}\right\} .
$$

Sejam $c\left(y_{k}^{j}\right)=c_{k}^{j}$ e $A y_{k}^{j}=a_{k}^{j}$. Temos que a reformulação do Problema Geral (3.1), com a restrição de convexidade para cada conjunto $S_{j}$, é dada por: 


$$
\begin{array}{ll}
\text { Maximizar } & \sum_{j=1}^{n} \sum_{k=1}^{p_{j}} c_{k}^{j} \lambda_{k}^{j} \\
\text { Sujeito } a & \sum_{j=1}^{n} \sum_{k=1}^{p_{j}} a_{k}^{j} \lambda_{k}^{j} \leq b \\
& \sum_{k=1}^{p_{j}} \lambda_{k}^{j}=1, \quad j=1, \ldots, n \\
& \lambda_{k}^{j} \in\{0,1\}, \quad j=1, \ldots, n, \quad k=1, \ldots, p_{j} .
\end{array}
$$

Como os subconjuntos são decompostos, cada conjunto $S_{j}^{*}$ apresenta sua lei de formação para a variável $y^{j}$. Neste caso, teremos $n$ subproblemas, onde cada $j$-ésimo subproblema é da forma

$$
\begin{array}{ll}
\text { Maximizar } & c\left(y^{j}\right)-\left(\pi^{T} A\right) y^{j}-\nu_{j} \\
\text { Sujeito a } & y^{j} \in S_{j}^{*}
\end{array}
$$

onde $(\pi, \nu)$, com $\pi \in R^{m}$ e $\nu \in R^{n}$, é o vetor multiplicador simplex e $j=1, \ldots, n$.

Se os subconjuntos $S_{j}$ são idênticos, isto é, $S_{j}=\bar{S}=\left\{y_{1}, \ldots, y_{p}\right\}$, para $j=1, \ldots, n$, então os $S_{j}$ podem ser representados por um único conjunto $\bar{S}$, e definindo $\lambda_{k}=\sum_{1 \leq j \leq n} \lambda_{k}^{j}$, a restrição de convexidade é substituída pela restrição $\sum_{1 \leq k \leq p} \lambda_{k}=n \operatorname{com} \lambda_{k} \geq 0$. Isto sugere o seguinte Problema Reformulado

$$
\begin{array}{ll}
\text { Maximizar } & \sum_{k=1}^{p} c_{k} \lambda_{k} \\
\text { Sujeito } a & \sum_{k=1}^{p} a_{k} \lambda_{k} \leq b \\
& \sum_{k=1}^{p} \lambda_{k}=n \\
& \lambda_{k} \geq 0 \text { e inteiro, } \quad k=1, \ldots, p .
\end{array}
$$

Dado o vetor multiplicador simplex $(\pi, \nu)$, com $\pi \in R^{m}$ e $\nu \in R$, o subproblema referente ao Problema Reformulado (3.7) é da forma

$$
\begin{array}{ll}
\text { Maximizar } & c(y)-\left(\pi^{T} A\right) y-\nu \\
\text { Sujeito a } & y \in \bar{S}
\end{array}
$$


O procedimento usado acima para a obtenção dos Problemas Reformulados (3.3), (3.5) e (3.7) é a utilização do Princípio da Decomposição de Dantzig e Wolfe em problemas de Otimização Linear Inteira.

Para maiores detalhes da Decomposição Dantzig e Wolfe em problemas de Otimização Linear, veja o Apêndice B. No Princípio da Decomposição de Dantzig e Wolfe em Problemas de Otimização Linear, os elementos do conjunto $S$ são representados por uma combinação convexa dos pontos de extremos e raios de $S$, e os coeficientes de convexidade não são necessariamente inteiros. Vanderbeck (2000) classifica que no caso do problema de Otimização Inteira, a decomposição consiste numa discretização do conjunto $S^{*}$ e para o problema de Otimização Linear, a decomposição consiste numa convexização de $S$.

Como o Problema Reformulado possui um número muito alto de colunas, torna-se necessário utilizar um conjunto restrito de colunas, sendo que colunas adicionais serão geradas quando necessário. O problema com um conjunto restrito de colunas é chamado de Problema Reformulado Restrito ou Problema Mestre Restrito.

\subsubsection{Problemas de Particionamento de Conjuntos}

Na última década, a técnica de geração de colunas para problemas de Otimização Inteira tem sido aplicada com muito sucesso em problemas que podem ser formulados como problemas de particionamento de conjuntos com variáveis binárias. No Problema de Partição de Conjunto, temos um conjunto de elementos e regras para gerar subconjuntos factíveis com os seus respectivos lucros. O problema consiste em determinar o lucro máximo ao se particionar o conjunto em subconjuntos factíveis. Seja $z_{i j}=1$ se o elemento $i$ está no subconjunto $j$ e 0 caso contrário. O Problema de Partição de Conjunto tem a forma

$$
\begin{array}{ll}
\text { Maximizar } & \sum_{j=1}^{n} c\left(z_{j}\right) \\
\text { Sujeito a } & \sum_{j=1}^{n} z_{i j}=1, \quad i=1, \ldots, m \\
& z_{j} \in S \\
& z_{i j} \in\{0,1\}
\end{array}
$$


onde $z_{j}=\left(z_{1 j}, z_{2 j}, \ldots, z_{m j}\right), m$ é o número de elementos no conjunto, $n$ é o número de subconjuntos e $S$ é o conjunto de subconjuntos factíveis.

Supondo inicialmente que os subconjuntos factíveis $S_{j}$ são dados por:

$$
S_{j}=\left\{z_{j}: D_{j} z_{j} \leq d_{j}, \quad z_{j} \in\{0,1\}^{m}\right\}
$$

o Problema de Particionamento de Conjunto (3.9) tem a seguinte forma:

$$
\begin{array}{ll}
\text { Maximizar } & \sum_{j=1}^{n} c_{j}\left(z_{j}\right) \\
\text { Sujeito a } & \sum_{j=1}^{n} z_{i j}=1, \quad i=1, \ldots, m \\
& D_{j} z_{j} \leq d_{j}, \quad j=1, \ldots, n \\
& z \in\{0,1\} .
\end{array}
$$

Sejam $S_{j}^{*}=\left\{y_{1}^{j}, y_{2}^{j}, \ldots, y_{p_{j}}^{j}\right\}, j=1, \ldots, n$ e $c_{j}^{k}=c_{j}\left(y_{k}^{j}\right)$. Então a reformulação do problema (3.11) é dado por:

$$
\begin{array}{ll}
\text { Maximizar } \quad & \sum_{j=1}^{n} \sum_{k=1}^{p_{j}}\left(c_{k}^{j} y_{k}^{j}\right) \lambda_{k}^{j} \\
\text { Sujeito a } \quad & \sum_{j=1}^{n} \sum_{k=1}^{p_{j}} y_{i k}^{j} \lambda_{k}^{j}=1, \quad i=1, \ldots, m \\
& \sum_{k=1}^{p_{j}} \lambda_{k}^{j} \leq 1, \quad j=1, \ldots, n \\
& \lambda_{k}^{j} \in\{0,1\}, \quad j=1, \ldots, n, \quad k=1, \ldots, p_{j} .
\end{array}
$$

Dado o vetor multiplicador simplex $(\pi, \nu)$, com $\pi \in R^{m}$ e $\nu \in R^{n}$, o j-ésimo subproblema de determinar a coluna com maior custo relativo é dado por:

$$
\begin{array}{ll}
\text { Maximizar } & c_{j}\left(y^{j}\right)-\pi^{T} y^{j}-\nu_{j} \\
\text { Sujeito a } & y^{j} \in S_{j}
\end{array}
$$

Supondo agora que os subconjuntos factíveis possua restrições idênticas. Então os conjuntos (3.10) podem ser substituídos por um único conjunto de inequações 


$$
\bar{S}=\left\{z_{j}: D z_{j} \leq d, z_{j} \in\{0,1\}\right\}
$$

e o Problema de Partição de Conjunto (3.9) tem a sua reformulação dada por:

$$
\begin{array}{ll}
\text { Maximizar } & \sum_{k=1}^{p} c_{k} \lambda_{k} \\
\text { Sujeito a } & \sum_{k=1}^{p} y_{i k} \lambda_{k}=1, \quad i=1, \ldots, m \\
& \lambda_{k} \in\{0,1\}, \quad k=1, \ldots, p .
\end{array}
$$

onde $c_{k}=c\left(y_{k}\right)$. A restrição de convexidade foi omitida, pois é comum nestes tipos de aplicações o valor de $n$ não ser fixo. Esta estrutura ocorre, por exemplo, em problemas de designação generalizada com máquinas idênticas e no problema binário de corte de estoque.

O subproblema referente ao Problema Reformulado (3.15) é dado por:

$$
\begin{array}{ll}
\text { Maximizar } & c(y)-\pi^{T} y-\nu \\
\text { Sujeito a } & y \in \bar{S}
\end{array}
$$

onde $(\pi, \nu)$, $\operatorname{com} \pi \in R^{m}$ e $\nu \in R$, é o vetor multiplicador simplex.

Apesar de termos discutido acima o problema de particionamento de conjunto, muitos modelos permitem que o Problema Reformulado seja modelado como problema de Cobertura de Conjuntos, onde as restriçôes de igualdade em (3.9) são do tipo $\geq$.

\subsection{O Problema Reformulado}

A relaxação por Otimização Linear de um problema de Otimização Inteira consiste em omitir as restrições de integralidade. O principal uso da relaxação em problemas de Otimização Inteira é que o valor ótimo do problema relaxado fornece um limitante inferior ou superior para o problema de Otimizaçāo Inteira, dependendo de que problema seja de minimizar ou maximizar a função objetivo, respectivamente. Além disso, se a solução ótima do problema relaxado for inteira, então ela também é solução ótima para o problema de Otimização Inteira. Se o problema relaxado é infactível, então o problema de Otimização Inteira também é infactível. 
Segundo Johnson et al. (2000), enquanto outras relaxações, tal como a Lagrangiana, são muito importantes em situaçōes especiais, a relaxação por Otimização Linear é ainda a principal em produzir limitantes para o problema de Otimização Inteira.

\subsubsection{Problema Geral Versus Problema Reformulado}

O Problema Geral (3.1) e o Problema Reformulado (3.3) são duas formulações de um mesmo problema. Ambas as formulações possuem o mesmo conjunto factível de soluções inteiras e, portanto, a mesma solução ótima. Entretanto, as duas formulações diferem em suas relaxações. Os limitantes fornecidos pelas relaxações do problema (3.1) e (3.3) são respectivamente,

$Z_{L P}(P G)=\operatorname{Maximizar}\left\{c^{T} x: A x \leq b, x \in S, x \geq 0\right\}$

$Z_{L P}(P R)=\operatorname{Maximizar}\left\{c^{T} x: A x \leq b, x \in \operatorname{conv}(x \in S\right.$ e $x$ inteiro $\left.)\right\}$

Uma solução fracionária da relaxação do Problema Geral (3.1) é uma solução factível para a relaxação do Problema Reformulado (3.3) se, e somente se, a solução fracionária possa ser representada por uma combinação convexa dos pontos extremos do $\operatorname{conv}\left(S^{*}\right)$.

Para esclarecer a afirmação acima vamos utilizar o seguinte exemplo. Supondo que a região de factibilidade para o Problema Geral (3.1) seja o conjunto $x \in S$ e $x$ inteiro. Neste caso os pontos factíveis para o Problema Geral (3.1) e para o Problema Reformulado (3.3) são dadas pela figura 3.1. 


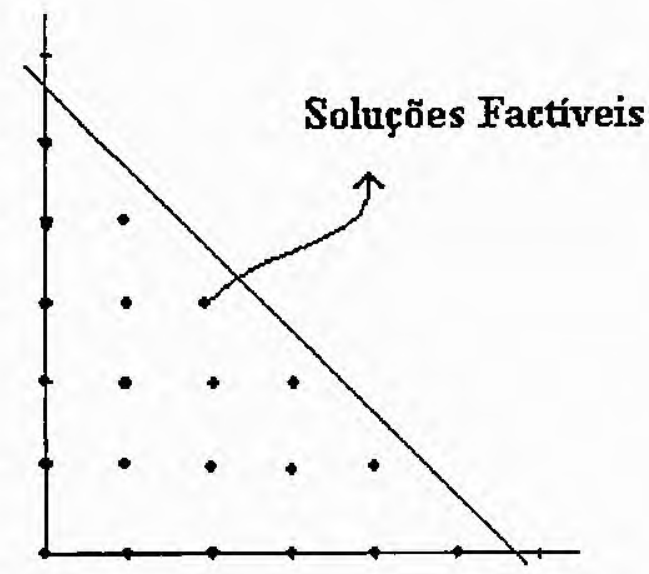

Figura 3.1: soluções Factíveis do Problema Geral e do Problema Reformulado

A região de factibilidade da relaxação do Problema Geral (3:1) é dada-pela figura 3.2.

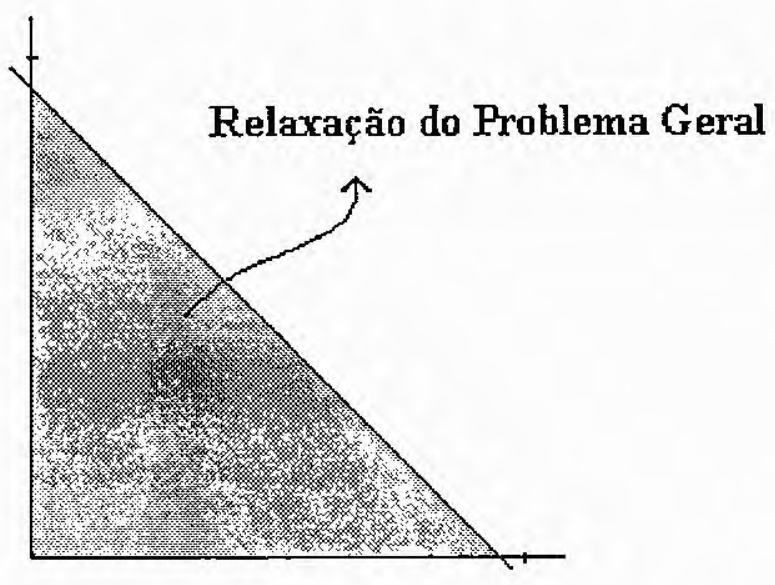

Figura 3.2: região de factibilidade da relaxação do Problema Geral

e a região de factibilidade da a relaxação do Problema Reformulado (3.3) é dada pela figura 3.3.

Considere a solução factível fracionária $x$ da relaxaçāo do Problema Geral (3.1) (figura $3.4)$.

Observe que a solução factível fracionária $x$ não é uma solução factível para a relaxação do Problema Reformulado (3.3). Então, podemos ter soluções factíveis para a relaxação do Problema Geral que nāo são soluções factíveis para a relaxação do Problema Reformulado. Assim, temos que 


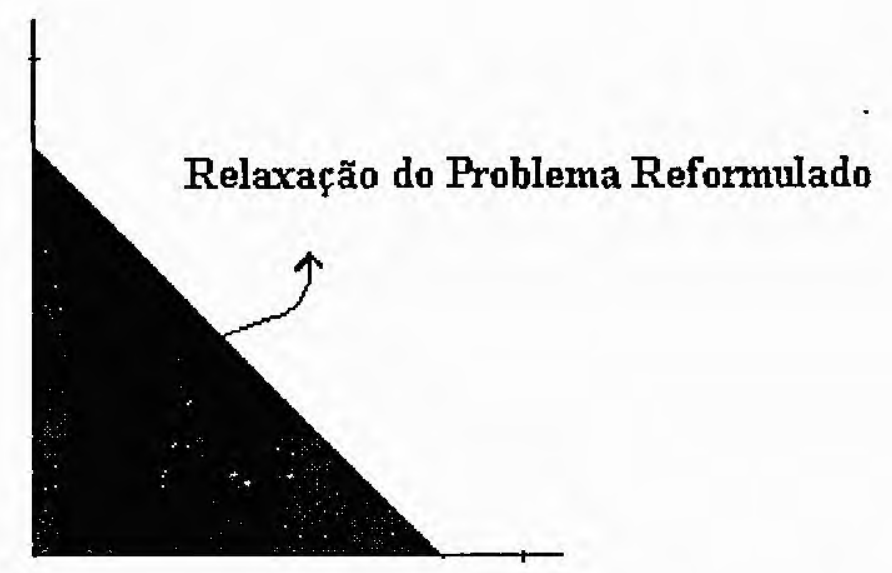

Figura 3.3: regiāo de factibilidade da relaxação do Problema Reformulado

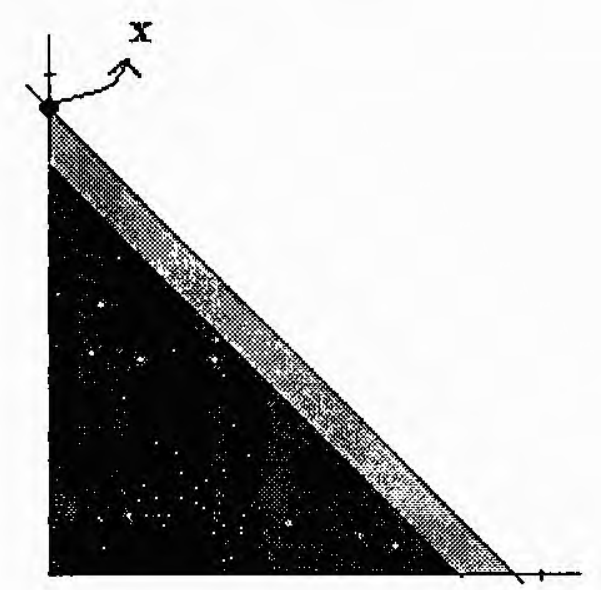

Figura 3.4: solução factível fracionária $x$ da relaxação do Problema Geral

$$
Z_{L P}(P G) \geq Z_{L P}(P R) \geq Z_{I P}
$$

onde $Z_{I P}$ é a solução ótima da função objetivo do Problema Geral. Embora a relaxação do Problema Reformulado possua um número muito grande de colunas, o modelo fornece um limitante melhor que a relaxação do Problema Geral. Este é um dos motivos para a utilização de formulações com número muito grande de colunas.

\subsubsection{Relaxação Lagrangiana}

A relaxação Lagrangiana é uma outra alternativa para se obter uma relaxação melhor para o problema de Otimização Inteira. 
A relaxação Lagrangiana do Problema Geral (3.1) é dada por (função dual):

$$
\begin{aligned}
g(\pi)= & \text { Maximizar }\left[c^{T} x+\pi^{T}(b-A x)\right] \\
& x \in S \\
& x \text { inteiro }
\end{aligned}
$$

$\operatorname{com} \pi \geq 0$.

Segue que $g(\pi) \leq c^{T} x$, para qualquer $x$ tal que $A x \leq b, x \in S$ e $x$ inteiro.

O problema dual Lagrangiano consiste em determinar o melhor dos limitantes inferiores, definido pela função dual $g(\pi)$ :

$$
g=\text { Minimizar }\{g(\pi), \pi \geq 0\}
$$

Geoffrion (1974) formulou um importante teorema que relaciona a relaxação Lagrangiana à relaxação do Problema Reformulado.

Teorema: $\mathrm{O}$ valor $g$ obtido da Relaxação Lagrangiana é igual ao valor máximo obtido pela relaxação do Problema Reformulado.

Prova: A função Lagrangiana dada por

$$
\begin{aligned}
g(\pi)= & \text { Maximizar }\left[c^{T} x+\pi^{T}(b-A x)\right] \\
& x \in S^{*}
\end{aligned}
$$

com $S^{*}=\{x \in S: x$ Inteiro $\}$ é equivalente a:

$$
\begin{aligned}
g(\pi)= & \text { Maximizar }\left[c^{T} x+\pi^{T}(b-A x)\right] \\
& x \in \operatorname{conv}\left(S^{*}\right)
\end{aligned}
$$

Deste modo

$$
\begin{aligned}
g & =\operatorname{Min}_{\pi \geq 0} g(\pi) \\
& =\operatorname{Min}_{\pi \geq 0} \operatorname{Max}_{x \in \operatorname{conv}\left(S^{*}\right)}\left[c^{T} x+\pi^{T}(b-A x)\right]
\end{aligned}
$$


Supondo o conjunto $S^{*}$ limitado, ou seja $S^{*}=\left\{y_{1}, \ldots, y_{p}\right\}$, então

$$
\operatorname{Max}_{x \in c o n v\left(S^{*}\right)}\left[c^{T} x+\pi^{T}(b-A x)\right]=c^{T} y_{k}+\pi^{T}\left(b-A y_{k}\right), \text { para algum } k .
$$

Assim

$$
\begin{aligned}
g= & \operatorname{Min}_{\pi}\left[\operatorname{Max} x_{1 \leq k \leq p} c^{T} y_{k}+\pi^{T}\left(b-A y_{k}\right)\right] \\
& \pi \geq 0
\end{aligned}
$$

o qual pode ser reescrito por

$$
\begin{aligned}
g= & \operatorname{Min}_{(\pi, \eta)} \eta \\
& \eta+\pi^{T}\left(A y_{k}-b\right) \geq c^{T} y_{k} \quad, k=1, \ldots, p \\
& \pi \geq 0 .
\end{aligned}
$$

Calculando o dual do problema acima (problema de otimização linear), obtemos:

$$
\begin{aligned}
g= & \text { Maximizar } c\left(\sum_{k=1}^{p} \lambda_{k} y_{k}\right) \\
& \sum_{k=1}^{p} \lambda_{k}=1 \\
& A\left(\sum_{k=1}^{p} y_{k} \lambda_{k}\right) \leq b\left(\sum_{k=1}^{p} \lambda_{k}\right) \\
& \lambda_{k} \geq 0, \quad k=1, \ldots, p .
\end{aligned}
$$

Definindo $c_{k}=c y_{k}$ e $a_{k}=A y_{k}$, temos que o problema

$$
\begin{aligned}
g= & \text { Maximizar } \sum_{k=1}^{p} c_{k} \lambda_{k} \\
& \sum_{k=1}^{p} \lambda_{k}=1 \\
& \sum_{k=1}^{p} a_{k} \lambda_{k} \leq b \\
& \lambda_{k} \geq 0, \quad k=1, \ldots, p
\end{aligned}
$$

corresponde precisamente à relaxação do Problema Reformulado. 
Temos também que o vetor multiplicador $\pi$ corresponde às variáveis duais da restrição

$$
\sum_{k=1}^{p}\left(A y_{k}\right) \lambda_{k} \leq b
$$

na relaxação do Problema Reformulado Restrito.

Então a escolha entre Relaxação Lagrangiana e o Problema Reformulado é a da abordagem que determina um valor ótimo mais eficiente para $\pi$. Segundo Barnhart et al. (1998), o método do subgradiente é uma técnica tradicional para se resolver a Relaxação Lagrangiana, para o qual a função objetivo é linear por partes. O algoritmo do subgradiente possui implementação fácil para as aplicações. Entretanto, o algoritmo pode ser lento e podem ocorrer problemas de convergência em problemas de grande escala. Outros métodos de otimização não diferenciáveis, tal como bundle methods, podem ser bem adequados, mas não foram suficientemente testados para problemas de otimização combinatória inteira. Durante os últimos 25 anos, o método de otimização do subgradiente tem sido muito usado. A utilizaçāo deste método foi motivada pela falta de códigos simplex eficientes com geração de colunas e a carência de memória computacional suficiente para o problema. Entretanto, esta situação tem mudado brutalmente com códigos simplex modernos. Essa mudança pode ser observada comparando os trabalhos de Savelsbergh (1997) e Guignard e Rosenwein (1989) sobre o problema de designação generalizada. A relaxação linear no trabalho de Savelsbergh (1997) claramente supera os métodos não lineares.

\subsection{O Algoritmo Branch and Price}

O algoritmo Branch and Price é um procedimento para resolver problemas de Otimização Inteira utilizando o método branch and bound. Em cada nó da árvore do branch and bound, a relaxação do Problema Reformulado é resolvida por geração de colunas. Embora - Problema Reformulado possua um número muito grande de variáveis, existem várias razōes para considerar essa formulação:

- A formulação original do problema de Otimização Inteira pode ter uma fraca relaxação por Otimização Linear. A relaxação pode ser melhorada utilizando reformulações que envolvam um número muito grande de variáveis, como o caso da Decom- 
posição Dantzig e Wolfe (parte da natureza combinatória do problema é considerada na reformulação).

- A formulação original do problema de Otimização Inteira pode ter estrutura simétrica, que faz o algoritmo branch and bound ser mal executado porque em cada ramificação dificilmente o problema é modificado. A reformulação com um número muito grande de variáveis pode eliminar essa simetria.

- A reformulação com um número muito grande de variáveis pode ser a única escolha.

A solução da relaxação do Problema Reformulado pode não ser inteira e, aplicando o algoritmo branch and bound sobre o Problema Reformulado Restrito, nāo é garantido encontrar a solução ótima ou mesmo factível. Após a ramificação, pode ser que existam colunas que possam melhorar a função objetivo, mas que não estejam presentes no Problema Reformulado Restrito. Então para encontrax a solução ótima, precisamos gerar colunas após a ramificação.

\subsection{Estratégias de Ramificação}

Ramificação refere-se ao processo de particionax o espaço de solução e eliminar a solução fracionária. A dificuldade de incorporar a geração de colunas ao branch and bound é que ramificações convencionais não são compatíveis com o subproblema, porque ao fixar variáveis, podemos gerar colunas que são infactíveis devido as restrições de ramificação e destruir a estrutura do subproblema, tornando-o mais difícil de ser resolvido. Recentemente, várias pesquisas tem sido desenvolvidas com o objetivo de determinar estratégias de ramificações que superem essas dificuldades, como por exemplo Desrochers et al. (1992) para roteamento de veículos, Desrochers e Soumis (1989) e Anbil et al. (1993) para problemas de planejamento de equipes, Vance et al. (1992) para problemas de corte de estoque e Savelbergh (1997) para problemas de designação generalizada.

Barnhart et al. (1998) apresentam estratégias de ramificação para problemas de Particionamento de Conjuntos e para problemas Gerais de Otimização Inteira, as quais serão descritas abaixo. 


\subsubsection{Problema de Partição de Conjunto}

Ryan and Foster (1981) propuseram uma estratégia de ramificação e embora não considerassem a técnica geração de colunas, esta regra é muito importante neste contexto. A estratégia é baseada na seguinte proposição:

Proposição 3.1. Se Y é uma matriz cujos elementos são 0 ou 1 e a solução básica do sistema $Y \lambda=1$ é fracionária, então existem duas linhas $r$ e $s$ do Problema Reformulado tais que

$$
0<\sum_{k: y_{r k}=1 e y_{s k}=1} \lambda_{k}<1
$$

Prova: Considere a variável fracionária $\lambda_{k}^{\prime}$. Seja $r$ uma linha com $y_{r k^{\prime}}=1$. Como $\sum_{1 \leq k \leq p} y_{r k} \lambda_{k}=1$ e $\lambda_{k^{\prime}}$ é fracionária, então existe uma outra coluna $k^{\prime \prime}$, com $0<\lambda_{k^{\prime \prime}}<1$ e $y_{r k^{\prime \prime}}=1$. Como não existem colunas iguais na base, deve existir uma linha $s$ tal que $y_{s k^{\prime}}=1$ ou $y_{s k^{\prime \prime}}=0$, mas nunca ambos. Então temos que

$$
\begin{aligned}
1 & =\sum_{1 \leq k \leq p} y_{r k} \lambda_{k} \\
& =\sum_{k: y_{r k}=1} \lambda_{k} \\
& >\sum_{k: y_{r k}=1 \mathrm{e} y_{s k}=1} \lambda_{k},
\end{aligned}
$$

onde a última somatória inclui ou $\lambda_{k^{\prime}}$ ou $\lambda_{k^{\prime \prime}}$ mas não ambos.

As linhas $r$ e $s$ fornecem o par de restrições de ramificação

$$
\sum_{k: y_{r k}=1 \mathrm{e}} \lambda_{k}=1 \text { e } \sum_{k: y_{r k}=1 \mathrm{e}} \lambda_{y_{s k}=1}=0
$$

ou seja, na primeira ramificação a linhas $r$ e $s$ precisam ser cobertas pelas mesmas colunas e por diferentes colunas na segunda ramificação.

A preposição implica que se nenhum par de ramificação pode ser obtido, então a solução para o problema mestre é inteira.

\section{Restrições Diferentes no Subconjunto}

Para este caso, se aplicarmos a estratégia de ramificação proposta por Ryan and Foster (1981) e selecionarmos uma linha referente à partição do conjunto e outra referente à 
restrição de convexidade, é obtida uma especial estratégia de ramificação que tem uma interpretação natural na formulação do Problema Original. Selecionando as referentes linhas, o par de restrições para a ramificação tem a forma:

$$
\sum_{1 \leq k \leq p_{s}: y_{\tau k}^{s}=1} \lambda_{k}^{s}=1 \quad \text { e } \sum_{1 \leq k \leq p_{s}: y_{\tau k}^{s}=1} \lambda_{k}^{s}=0 .
$$

Esta estratégia de ramificação exige que o elemento $r$ esteja no conjunto $s$ na ramificação à esquerda e que o elemento $r$ esteja em qualquer conjunto, com exceção do conjunto $s$, na ramificação à direita. Esta ramificação tem uma interpretação natural, pois corresponde precisamente à ramificação usual no Problema de Partição de Conjunto (3.11), uma vez que

$$
\sum_{1 \leq k \leq p_{s}: y_{\tau k}^{s}=1} \lambda_{k}^{s}=1 \Rightarrow \sum_{1 \leq k \leq p_{s}} y_{\tau k}^{s} \lambda_{k}^{s}=1 \Rightarrow z_{i j}=1
$$

e

$$
\sum_{1 \leq k \leq p_{s}: y_{r k}^{s}=0} \lambda_{k}^{s}=0 \Rightarrow \sum_{1 \leq k \leq p_{s}} y_{r k}^{s} \lambda_{k}^{s}=0 \Rightarrow z_{i j}=0
$$

Como a estratégia de ramificação do problema (3.11) representa a ramificação usual do problema (3.12), o que diferencia os dois problemas são as suas formulações.

\section{Restrições Idênticas no Subconjunto}

A estratégia de ramificação sugerida por Ryan and Foster (1981) exige que os elementos $r$ e $s$ pertençam ao mesmo subconjunto na ramificação à esquerda e para diferentes conjuntos na ramificação à direita. Então para a ramificação à esquerda, todas as colunas factíveis precisam ter $y_{\tau k}=y_{s k}=0$ ou $y_{r k}=y_{s k}=1$, enquanto na ramificação à direita, todas as colunas factíveis precisam ter $y_{r k}=y_{s k}=0$ ou $y_{r k}=0, y_{s k}=1$ ou $y_{r k}=1, y_{s k}=0$. Ao adicionarmos restrições de ramificação, novas variáveis do vetor multiplicador simplex serāo introduzidas no subproblema. Ao invés de adicionarmos restrições de ramificações explicitamente no Problema Reformulado, as colunas infactíveis do Problema Reformulado podem ser eliminadas. A ramificação à esquerda equivale a combinar as linhas $r$ e $s$ no Problema Reformulado, obtendo um problema de particionamento de conjunto menor. Na ramificação à direita, as linhas $r$ e $s$ são disjuntas, que pode produzir um problema de particionamento de conjunto mais fácil e colunas disjuntas 
favorecem o surgimento de soluções inteiras. Não adicionar explicitamente restrições de ramificação no Problema Reformulado, tem a vantagem de não incluir novas variáveis do vetor multiplicador simplex no subproblema.

Forçando dois elementos a estarem no mesmo subconjunto em uma ramificação e forçando dois elementos estarem em subconjuntos distintos na outra ramificação, é bastante fácil de se executar a ramificação. Entretanto, o subproblema em uma ramificação pode ser mais complicado que na outra ramificação.

\subsubsection{Problema Geral Reformulado}

Discutimos anteriormente as estratégias de ramificação para o problema de partição de conjuntos. Agora, vamos verificar as estratégias de ramificação para o Problema Geral Reformulado (3.3).

\section{Restrições Diferentes no Subconjunto}

Segundo Johnson (1989), a estratégia de ramificação para o Problema Reformulado Geral Inteiro, com diferentes restriçōes de subconjunto, pode ser obtida diretamente da exigência de integralidade das variáveis originais $x_{i j}$ do problema (3.1). A solução ótima para o Problema Geral (3.1) é infactível se, e somente se

$$
x_{j}=\sum_{k=1}^{p_{j}} y_{k}^{j} \lambda_{k}^{j}
$$

tem alguma componente $r$ fracionária para algum $j$. Suponha que o valor fracionário seja igual a $\alpha$. Então é realizada o seguinte: em uma ramificação teremos

$$
\sum_{k=1}^{p_{j}} y_{k}^{j} \lambda_{k}^{j} \leq\lfloor\alpha\rfloor
$$

e para a outra ramificação teremos

$$
\sum_{k=1}^{p_{j}} y_{k}^{j} \lambda_{k}^{j} \geq\lceil\alpha\rceil
$$

\section{Restrições Idênticas no Subconjunto}

O desenvolvimento de estratégias de ramificação para o Problema Reformulado Geral Inteiro com idênticas restrições de subconjunto é mais complicado porque não queremos 
ramificar a partir das variáveis originais devido ao problema de simetria. No Problema de Partição de Conjuntos, a estratégia de ramificação proposta por Ryan e Foster (1981) sempre identificava um par de restrições de ramificação. Para o Problema Reformulado Geral, o par de restrições de ramificação também pode ser encontrado.

Considere $\lambda^{*}$ a solução ótima da relaxação do problema 3.7. Segundo Barnhart et al. (1998), se soluçāo $\lambda^{*}$ é fracionária, então sempre é possível identificar uma linha $r$ e um inteiro $\alpha_{r}$, tal que

$$
\sum_{k:\left(a_{k}\right)_{r} \geq \alpha_{r}} \lambda_{k}=\beta_{r}
$$

onde $\beta_{r}$ é fracionário.

Temos então duas restrições de ramificação:

$$
\sum_{k:\left(a_{k}\right)_{r} \geq \alpha_{r}} \lambda_{k}=\left\lfloor\beta_{r}\right\rfloor \text { e } \sum_{k:\left(a_{k}\right)_{r} \geq \alpha_{r}} \lambda_{k}=\left\lceil\beta_{r}\right\rceil
$$

Para o Problema de Partição de Conjuntos, as restrições de ramificação não eram acrescentadas explicitamente no Problema Reformulado, pois tais ramificações eram equivalentes a eliminar variáveis do subproblema. No Problema Geral Reformulado, geralmente as restrições de ramificação 3.17 não podem ser usadas para eliminar variáveis e, neste caso, precisam ser adicionadas explicitamente na relaxação do problema 3.18.

Se em cada nó $u$ da árvore forem adicionadas restrições de ramificação do tipo 3.17, temos que a relaxação do Problema Reformulado no nó $u$ tem a seguinte forma:

$$
\begin{aligned}
\text { Maximizar } \quad & \sum_{k=1}^{p} c_{k} \lambda_{k} \\
\text { Sujeito } a \quad & \sum_{k=1}^{p} a_{k} \lambda_{k} \leq b \\
& \sum_{k=1}^{p} \lambda_{k}=n \\
& \sum_{k:\left(a_{k}\right)_{r} \geq \alpha_{r}} \lambda=\left\lfloor\beta_{r}\right\rfloor, \quad r \in G^{u} \\
& \sum_{k:\left(a_{k}\right)_{r} \geq \alpha_{r}} \lambda=\left\lceil\beta_{r}\right\rceil, \quad r \in H^{u} \\
& \lambda_{k} \geq 0, \quad k=1, \ldots, p .
\end{aligned}
$$


onde $G^{u}$ e $H^{u}$ são os conjuntos de índices das restrições de ramificação. Cada $r \in G^{u} \mathrm{e}$ $r \in H^{u}$ corresponde aos pares $\left(\alpha_{r},\left\lfloor\beta_{r}\right\rfloor\right)$ e $\left(\alpha_{r},\left\lceil\beta_{r}\right\rceil\right)$, respectivamente.

Cada restrição de ramificação irá contribuir com um novo elemento do vetor multiplicador simplex no subproblema, se para a coluna $a$ gerada tivermos $(a)_{r} \geq \alpha_{r}, \operatorname{com} r \in G^{u}$ ou $r \in H^{u}$. Isto pode tornar o subproblema mais complicado.

Uma única linha $r$ pode não ser suficiente para definir as restrições de ramificação. Considere uma solução fracionária do Problema de Partição de Conjunto. O único valor possível para $\alpha_{r}$ é 1 . Entretanto, sempre teremos $\sum_{k: y_{k r} \geq 1} \lambda_{k}=1$ para toda linha. Podemos ter que ramificar em múltiplas linhas.

Seja

$$
\sum_{k:\left(a_{k}\right)_{r} \geq \alpha_{r}} \lambda_{k}=\beta_{r}
$$

$\operatorname{com} \beta_{r}$ inteiro para qualquer linha $r$ e inteiro $\alpha_{r}$. Para obter as restrições de ramificação, escolhemos uma linha qualquer, digamos $r$, e procuramos outra linha $s$ e inteiro $\alpha_{s}$, tal que

$$
\sum_{k:\left(a_{k}\right)_{r} \geq \alpha_{\tau} \text { e }\left(a_{k}\right)_{s} \geq \alpha_{s}} \lambda_{k}=\beta_{s}
$$

e $\beta_{s}$ fracionário. Se tais linhas existirem, temos as restrições de ramificação:

$$
\sum_{k:\left(a_{k}\right)_{r} \geq \alpha_{r} \text { e }\left(a_{k}\right)_{s} \geq \alpha_{s}} \lambda_{k}=\left\lfloor\beta_{s}\right\rfloor \text { e } \sum_{k:\left(a_{k}\right)_{r} \geq \alpha_{r} \mathrm{e}\left(a_{k}\right)_{s} \geq \alpha_{s}} \lambda_{k}=\left\lceil\beta_{s}\right\rceil
$$

Caso contrário, procuramos uma terceira linha.

\subsubsection{Problema de Designação Generalizada}

Para exemplificar as características das estratégias de ramificação do algoritmo Branch and Price, utilizaremos o problema de Designação Generalizada, cuja formulação é dado por Partição de Conjuntos. Como visto no Capítulo 2, a formulação padrão em Otimização Inteira do problema Designação Generalizada é a seguinte: 


$$
\begin{array}{ll}
\text { Maximizar } & \sum_{i=1}^{m} \sum_{j=1}^{n} p_{i j} z_{i j} \\
\text { Sujeito a } \quad & \sum_{j=1}^{n} z_{i j}=1, \quad i=1, \ldots, m \\
& \sum_{i=1}^{m} w_{i j} z_{i j} \leq d_{j}, \quad j=1, \ldots, n \\
& z_{i j} \in\{0,1\}, \quad i=1, \ldots, m, \quad j=1, \ldots, n .
\end{array}
$$

onde $p_{i j}>0$ é o desempenho da máquina $i$ em realizar a tarefa $j, w_{i j}>0$ é a capacidade da máquina necessária para realizar a tarefa $j$ na máquina $i, d_{j}$ é capacidade da máquina $j$ e $x_{i j}$ a variável binária que indica se a tarefa $i$ é designada para a máquina $j\left(x_{i j}=1\right)$ ou não $\left(x_{i j}=0\right)$.

Considerando $K^{j}=\left\{y_{1}^{j}, y_{2}^{j}, \ldots, y_{K_{j}}^{j}\right\}$ como conjunto de todas as possíveis designações de tarefas para a máquina $j$, onde $y_{k}^{j}=\left(y_{1 k}^{j}, y_{2 k}^{j}, \ldots, y_{m k}^{j}\right)$ satisfaz a restrição:

$$
\begin{aligned}
& \sum_{i=1}^{n} w_{i j} y_{i k}^{j} \leq d_{j} \\
& y_{i k}^{j} \in\{0,1\}, \quad i=1, \ldots, m .
\end{aligned}
$$

temos o Problema Reformulado:

$$
\begin{aligned}
\text { Maximizar } \quad & \sum_{j=1}^{n} \sum_{k=1}^{K_{j}}\left(\sum_{i=1}^{m} p_{i j} y_{i k}^{j}\right) \lambda_{k}^{j} \\
\text { Sujeito a } \quad & \sum_{j=1}^{n} \sum_{k=1}^{K_{j}} y_{i k}^{j} \lambda_{k}^{j}=1, \quad i=1, \ldots, m \\
& \sum_{k=1}^{K_{j}} \lambda_{k}^{j}=1, \quad j=1, \ldots, n \\
& \lambda_{k}^{j} \in\{0,1\}, \quad i=1, \ldots, m, \mathrm{~J}=1, \ldots, n .
\end{aligned}
$$

Seja $\lambda$ uma soluçāo da relaxação do Problema Reformulado 3.20. Como o Problema Original 3.19 e o Problema Reformulado 3.20 são equivalentes, dada a solução $\lambda$ do problema 3.19, a solução do problema 3.19 é dada por $z_{i j}=\sum_{k=1}^{K_{j}} y_{i k}^{j} \lambda_{k}^{j}$. Assim, dada uma solução do Problema Reformulado, temos uma correspondente ao Problema Original. 
A solução da relaxação do Problema Reformulado 3.20 resolvido por geração de colunas pode não ter solução inteira. Aplicando o algoritmo branch and bound ao Problema Reformulado Restrito num subconjunto de colunas, pode-se não encontrar uma solução ótima ou nem mesmo factível. Neste caso é necessário gerar colunas adicionais.

A ramificação padrão na variável $\lambda$ do problema reformulado 3.20 pode causar problemas quando alguma variável $\lambda_{k}^{j}$ for fixado em $\lambda_{k}^{j}=0$. Temos que $y_{k}^{j}$ representa uma solução particular do $j$-ésimo problema da mochila. Então $\lambda_{k}^{j}=0$ significa que esta solução é excluída. Entretanto é possível, e muito comum, que na próxima resolução do problema da mochila, a solução ótima seja precisamente aquela representada por $y_{k}^{j}$. Neste caso, torna-se necessário encontrar a segunda melhor solução para o problema da mochila. No nível $l$ da árvore do branch and bound, podemos ter que determinar a l-ésima melhor solução.

Para superar essa dificuldade, ao invés de ramificar na variável $\lambda^{\prime} s$ do Problema Reformulado, utilizamos a regra de ramificação sugerida por Ryan and Foster (1981) nas variáveis $z_{i j}$ do Problema Original. Selecionando uma linha $r$, referente à partição do conjunto, e outra linha $s$, referente à restrição de convexidade, temos que as restrições de convexidade

e

$$
\sum_{1 \leq k \leq p_{s}: y_{\tau k}^{s}=1} \lambda_{k}^{s}=1
$$

$$
\sum_{1 \leq k \leq p_{s}: y_{r k}^{s}=1} \lambda_{k}^{s}=0
$$

equivalem a

$$
z_{\text {rs }}=1 \text { e } z_{i j}=0
$$

respectivamente.

Quando $z_{r s}=1$, todas as colunas existentes no problema reformulado que não designam a tarefa $r$ para a máquina $s$ são apagadas e a tarefa $r$ é permanentemente designada para a máquina $s$, ou seja, a variável $y_{r}^{s}$ é fixada no valor 1 no $j$-ésimo problema da mochila.

Quando $z_{r s}=0$, todas as colunas existentes no problema reformulado que designam a tarefa $r$ para a máquina $s$ são apagadas e a tarefa $r$ não pode ser designada para a máquina $s$, ou seja, a variável $y_{\tau}^{s}$ é removida do $j$-ésimo problema da mochila. Para cada 
ramificação realizada, o problema da mochila contém uma variável a menos.

Para o Problema de Designação Generalizada, podemos ter o caso em que as máquinas são todas idênticas. Considerando as máquinas idênticas teremos $p_{i 1}=p_{i 2}=\ldots=p_{\text {in }}=$ $p_{i}$ e $w_{i 1}=w_{i 2}=\ldots=w_{i n}=w_{i}$, para $i=1, \ldots, m$.

Assim, o modelo de Designação Generalizada com máquinas idênticas é dado por:

$$
\begin{array}{ll}
\text { Maximizar } \quad & \sum_{i=1}^{m} \sum_{j=1}^{n} p_{i} z_{i j} \\
\text { Sujeito a } \quad & \sum_{j=1}^{n} z_{i j}=1, \quad i=1, \ldots, m \\
& \sum_{i=1}^{m} w_{i} z_{i j} \leq d, \quad j=1, \ldots, n \\
& z_{i j} \in\{0,1\}, \quad i=1, \ldots, m, \quad j=1, \ldots, n .
\end{array}
$$

Um detalhe muito importante relacionado com o problema (3.21) é relativo à simetria. Esta simetria pode causar ramificações muito "pobres"nas variáveis $z_{i j}$. Com máquinas idênticas, existe um número exponencial de soluções que diferem somente pelos nomes $j$ da máquina. Vamos verificar um exemplo disso. Suponha que para o problema (3.21) o número de máquinas seja $n=3$, o número de tarefas $m=4$ e que uma solução seja dada por 


$$
z=\left(\begin{array}{c}
z_{11} \\
z_{21} \\
z_{31} \\
z_{41} \\
\cdots \\
z_{12} \\
z_{22} \\
z_{32} \\
z_{42} \\
\cdots \\
z_{13} \\
z_{23} \\
z_{33} \\
z_{43}
\end{array}\right)=\left(\begin{array}{c}
1 \\
0 \\
0 \\
0 \\
\cdots \\
0 \\
1 \\
0 \\
0 \\
\cdots \\
0 \\
0 \\
1 \\
1
\end{array}\right)
$$

Esta solução designa as tarefas 1 e 2 a duas máquinas (neste caso, máquinas 1 e 2) e as tarefas 3 e 4 a uma máquina (máquina 3).

Como as máquinas são idênticas, as seguintes soluções

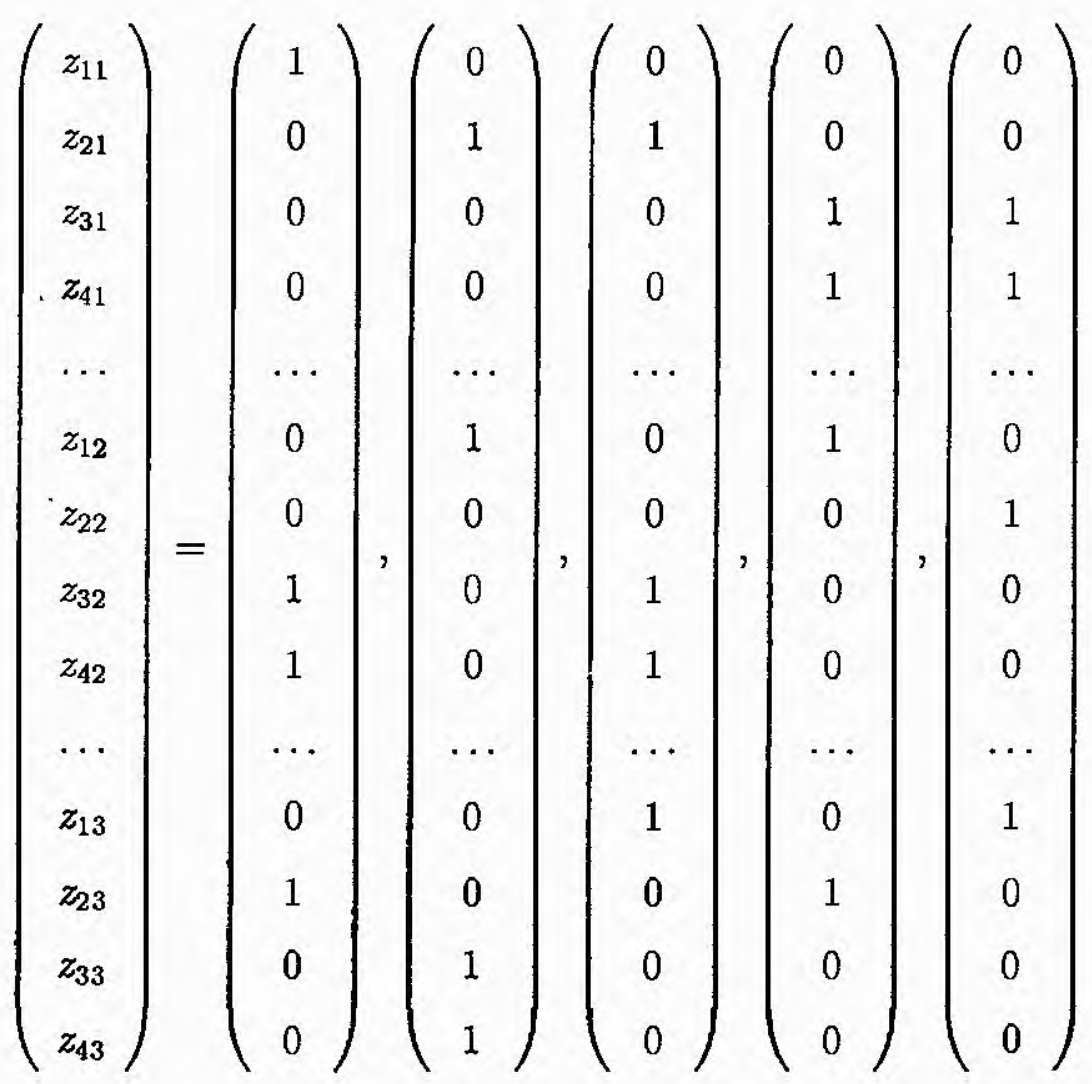


embora do ponto de vista do modelo sejam diferentes, representam as mesmas soluções. Ou seja, permutando as designações de duas máquinas, obtemos duas soluções que são diferentes em termos do modelo, mas que possuem o mesmo valor da função objetivo. Tais soluções são chamadas simétricas.

O problema de simetria novamente surge quando utilizamos o procedimento de branch and bound na relaxação do problema (3.21). Quando uma solução fracionária é excluída por uma ramificação, outra simétrica é gerada. Considerando ainda o exemplo anterior, suponha que tenhamos a solução para a relaxação do problema (3.21)

$$
z=\left(\begin{array}{c}
z_{11} \\
z_{21} \\
z_{31} \\
z_{41} \\
\ldots \\
z_{12} \\
z_{22} \\
z_{32} \\
z_{42} \\
\cdots \\
z_{13} \\
z_{23} \\
z_{33} \\
z_{43}
\end{array}\right)=\left(\begin{array}{c}
1 \\
0,3 \\
0 \\
0 \\
\ldots \\
0 \\
0,7 \\
0 \\
0 \\
\cdots \\
0 \\
0 \\
1 \\
1
\end{array}\right)
$$

Aplicando a ramificação padrão na variável fracionária $z_{21}=0,3$, teremos duas ramificações:

$$
z_{21}=0 \text { e } z_{21}=1
$$

Para $z_{21}=0$, pode ser que as soluções 


$$
z_{1}=\left(\begin{array}{c}
0 \\
0 \\
1 \\
1 \\
0 \\
0,7 \\
0 \\
0 \\
1 \\
0,3 \\
0 \\
0
\end{array}\right) \text { ou } z_{2}=\left(\begin{array}{c}
0 \\
0 \\
1 \\
1 \\
1 \\
0,3 \\
0 \\
0 \\
0 \\
0,7 \\
0 \\
0
\end{array}\right)
$$

apareça em algum lugar da árvore e que seja simétrica à anterior. Neste caso o valor fracionário aparece novamente numa outra variável, de tal forma que os valores da função objetivo fornecidos por $z, z_{1}$ e $z_{2}$ sejam respectivamente os mesmos e o procedimento branch and bound não consiga podar essas soluções simétricas.

Para evitar o problema de simetria utilizamos a reformulação do problema (3.21).

Considerando $K=\left\{y_{1}, y_{2}, \ldots, y_{K}\right\}$ como conjunto de todas as possíveis designações . de tarefas para a máquina idêntica, onde $y_{k}=\left(y_{1 k}, y_{2 k}, \ldots, y_{m k}\right)$ satisfaz à restrição:

$$
\begin{aligned}
& \sum_{i=1}^{n} w_{i j} y_{i k} \leq d \\
& y_{i k} \in\{0,1\}, \quad i=1, \ldots, m .
\end{aligned}
$$

Para este problema, como todas as máquinas são iguais, o problema da mochila será o mesmo para ambas as máquinas. Assim teremos que resolver apenas um subproblema. A variável $\lambda_{k}^{j}$ pode ser agregada, definindo $\sum_{j=1}^{n} \lambda_{k}^{j}=\lambda_{k}$ e as restrições de convexidade podem ser substituídas numa única restrição

$$
\sum_{k=1}^{K} \lambda_{k}=n
$$

$\lambda_{k}$ inteiro. 
Desta forma, a reformulação do problema (3.21) é dada por:

$$
\begin{array}{ll}
\text { Maximizar } & \sum_{k=1}^{K}\left(\sum_{i=1}^{m}\left(p_{i} y_{i k}^{j}\right) \lambda_{k}\right) \\
\text { Sujeito a } & \sum_{k=1}^{K} y_{i k}^{j} \lambda_{k}^{j}=1, \quad i=1, \ldots, m \\
& \sum_{k=1}^{K} \lambda_{k}=n, \quad j=1, \ldots, n \\
& \lambda_{k} \text { inteiro, } \quad k=1, \ldots, K .+
\end{array}
$$

Para resolver este problema, consideremos as linhas do Problema Reformulado com relação às tarefas $r$ e $s$. A ramificação é feita dividindo o espaço solução em um conjunto no qual $r$ e $s$ apareçam juntas e possam ser combinadas numa única tarefa no problema da mochila, e num outro conjunto no qual as tarefas precisarem aparecer separadamente e uma simples restrição do tipo

$$
y_{i r}+y_{i s} \leq 1
$$

é adicionada na mochila. 


\section{Capítulo 4}

\section{Pr blema de Roteamento com}

\section{Janela de Tempo}

\subsection{Introdução}

Roteamento e programação de veículos são atividades muito importantes em muitos sistemas de distribuição e transporte. Uma rota é uma seqüencia de pontos de carregamento ou descarregamento, denominados clientes, que o veículo deverá percorrer, de forma que inicie e termine o processo em um depósito ou domicílio. A programação de veículos consiste em determinar rotas, à quais estão associados instantes de chegada e partida de veículos, em cada cliente ou depósito.

O Problema de Roteamento é definido como o problema de visitar uma série de clientes com demandas conhecidas, com o menor custo possível e atendendo às eventuais imposições do problema. Cada veículo inicia e termina seu trajeto a partir de um depósito central e cada cliente é atendido exatamente uma vez, sendo a capacidade do veículo respeitada.

No Problema de Roteamento com Janela de Tempo cada cliente tem associado um intervalo de tempo $\left[a_{i}, b_{i}\right]$ que determina o tempo inicial e final que pode ser atendido.

O primeiro problema de roteamento estudado foi o Problema do Caixeiro Viajante. $\mathrm{O}$ Problema do Caixeiro Viajante consiste em encontrar uma sequência de cidades a serem visitadas por um caixeiro viajante, sendo cada cidade visitada exatamente uma vez e a distância total percorrida minimizada. Segundo Bodin et al. (1983), a primeira menção conhecida do problema é devido a Hassler Whitney em 1934 num trabalho na Princeton 


\section{University.}

Bodin et al. (1983) fizeram um trabalho que retratou estado-da-arte dos modelos de roteirização e programação de veículos e tripulações. Ainda hoje é considerado umas das importantes referências sobre o assunto, pois são considerados inúmeros tipos de problemas.

Os problemas de roteirização pertencem à classe $N P$-hard, ou seja, são problemas cujo esforço computacional para a sua resolução cresce exponencialmente com o seu tamanho, que neste caso é o número de nós.

O modelo a ser trabalhado nesta dissertação é o proposto por Desrochers et al. (1984). Esse artigo é um dos primeiros traballhos na literatura onde se utiliza o método Branch and Price. Desrochers et al. (1984) modelaram o problema como Problema de Partição de Conjunto e as colunas são geradas resolvendo o Problema do Caminho Mínimo com Janela de Tempo.

\subsection{Formulação Matemática}

Considere $P$ o conjunto de clientes da rede. Para o problema estudado, vamos considerar um único depósito de onde cada veículo parte uma única vez. O nós $s$ e $t$ representam a saída e a entrada no depósito, respectivamente. Assim, temos a rede $G=(N, A)$, onde $N=P \cup\{s, t\}$ é o conjunto de nós e $A=I \cup(\{s\} \times P) \cup(\{t\} \times P) \circ$ conjunto de arcos do tipo $(i, j)$ indicando que o nó $j$ pode ser visitado após o nó $i$. A cada arco $(i, j) \in A$ está associado um custo $c_{i j}$ e um tempo de duração $t_{i j}$ e, para cada nó $i \in N$ está associada uma janela de tempo $\left[a_{i}, b_{i}\right]$ que indica o tempo em que o veículo precisa chegar ao nó $i$. Se o veículo chegar no nó $i$ antes do tempo $a_{i}$, fica esperando até completar o tempo. Desrochers et al. (1984) consideram que cada nó $i \in P$ também pode representar uma rota pequena dentro do próprio nó (viagem) e os arcos entre os nós são chamados "inter-viagens".

Para a formulação matemática do problema utilizaremos as seguintes variáveis: $x_{i j}$, $(i, j) \in A$, a variável de fluxo binária tomando valor 1 quando o arco $(i, j)$ é percorrido pelo veículo e 0 caso contrário, e a variável de tempo $t_{i}, i \in P$, designando o instante de atendimento no nó $i$.

O Problema de Roteamento com Janela de Tempo pode ser formulado como o seguinte 
problema de programação inteira não linear:

$$
\begin{array}{ll}
\text { Minimizar } & \sum_{(i, j) \in A} c_{i j} x_{i j} \\
\text { Sujeito a } & \sum_{j \in N} x_{i j}=\sum_{j \in N} x_{j i}=1 \quad i \in P \\
& x_{i j} \geq 0 \quad(i, j) \in A \\
& x_{i j}>0 \Rightarrow t_{i}+t_{i j} \leq t_{j} \quad(i, j) \in N \\
& a_{i} \leq t_{i} \leq b_{i} \quad i \in P \\
& x_{i, j} \in\{0,1\} \quad(i, j) \in A
\end{array}
$$

A função objetivo 4.1 minimiza a soma dos custos dos arcos. A restrição 4.2 define a restrição de fluxo da rede para a rota que começa em $t$ e termina em $s$. A restrição 4.4 descreve a relação necessária entre rotas e programação, enquanto a restrição 4.5 estabelece a janela de tempo de cada nó.

Considerando somente as restrições 4.1, 4.2 e 4.3, temos o problema de roteamento sem as restrições de programação de horários, cuja solução é inteira e foi primeiramente estudado por Dantzig e Fulkerson (1954).

Observe que a restrição 4.4 não é uma restrição linear e pode ser escrita pela seguinte forma linear:

$$
t_{i}+t_{i j}-t_{j} \leq\left(1-x_{i j}\right) M_{i j}, \quad(i, j) \in I
$$

com $M_{i j} \geq b_{i}+t_{i j}-a_{j}$. Esta formulação exige que a variável $x_{i j}$ seja inteira, pois se relaxarmos a integralidade da variável $x_{i j}$, teremos

$$
0<t_{i}+t_{i j}+t_{j} \leq\left(1-x_{i j}\right) M_{i j}
$$

o que não satisfaz a restrição (4.4) do problema inicial. Portanto a variável $x_{i j}$ tem que ser inteira.

\subsection{Formulação por Partição de Conjunto}

O Problema de Roteamento com Janela de Tempo pode ser formulado como Partição de Conjunto. Para esta formulação, sejam: 
$R$ : o conjunto de rotas satisfazendo as restrições 4.4 e 4.5;

$\delta_{r i}$ : a constante binária que toma valor igual a 1 se a rota $r \in R$ inclui a viagem $i \in P$ e 0 caso contrário;

$c_{r}$ : custo da rota $r \in R$;

$y_{r}$ : a variável binária que toma valor igual a 1 se a rota $r \in R$ é utilizada e 0 caso contrário.

A formulação do problema por partição de conjunto que determina as rotas com menor custo satisfazendo as restrições de programação e incluindo cada nó exatamente uma vez, é dada por:

$$
\begin{array}{lll}
\text { Minimizar } & \sum_{r \in R} c_{r} y_{r} \\
\text { Sujeito a } & \sum_{r \in R} \delta_{r i} y_{r}=1, \quad i \in P \\
& y_{r} \in\{0,1\}, \quad r \in R .
\end{array}
$$

A restrição 4.9 diz que dentre todas as rotas existentes, todos os nós serão incluídos exatamente uma vez. As colunas representadas por $\delta_{r}$ correspondem a rotas factíveis. Como o número de rotas factíveis é extremamente grande, o problema não pode ser resolvido diretamente, ou seja, não podemos explicitar todas as colunas do problema. Em vez disso, é possível resolver a relaxação do Problema de Partição de Conjuntos pela técnica de geração de colunas. Colunas factíveis serão geradas, quando necessário, resolvendo um subproblema.

Uma nova rota será gerada calculando o menor dos custos relativos. Seja $\delta_{t}=$ $\left(\delta_{t 1}, \delta_{t 2}, \ldots, \delta_{t n}\right)$ uma coluna representando uma rota qualquer. O custo relativo da coluna $\delta_{t}$ é dado por:

$$
c_{t}-\pi^{T} \delta_{t}
$$

onde $\pi$ é o vetor multiplicador simplex.

Como $\delta_{t 1}=1$ indica se a rota passa pelo nó $i$ e $\delta_{t 1}=0$ caso contrário, temos que: 


$$
\begin{gathered}
\delta_{t 1}=\sum_{j:(i, j) \in A} x_{j 1}, x_{j 1}=\{0,1\} \text { para } j \text { tal que }(i, j) \in A, \\
\delta_{t 2}=\sum_{j:(i, j) \in A} x_{j 2}, x_{j 2}=\{0,1\} \text { para } j \text { tal que }(i, j) \in A, \\
\vdots \\
\delta_{t n}=\sum_{j:(i, j) \in A} x_{j n}, \quad x_{j n}=\{0,1\} \text { para } j \text { tal que }(i, j) \in A .
\end{gathered}
$$

Assim o custo da coluna $\delta_{t}, c_{t}$, será dado por:

$$
c_{t}=\sum \sum_{(i, j) \in A} c_{i j} x_{i j}
$$

Temos então que $c_{t}$ será:

$$
\begin{aligned}
c_{t}-\pi^{T} \delta_{t} & =\sum \sum_{(i, j) \in A} c_{i j} x_{i j}-\sum_{i \in P} \sigma_{i} \delta_{t i} \\
& =\sum \sum_{(i, j) \in A} c_{i j} x_{i j}-\sum \sum_{(i, j) \in A} \sigma_{i} x_{i j} \\
& =\sum \sum_{(i, j) \in A}\left(c_{i j}-\sigma_{i}\right) x_{i j}
\end{aligned}
$$

Portanto, a coluna com o menor custo relativo será gerada resolvendo o Problema do Caminho Mínimo com Janela de Tempo:

$$
\begin{aligned}
\text { Minimizar } & \sum \sum_{(i, j) \in A}\left(c_{i j}-\sigma_{i}\right) x_{i j} \\
\text { Sujeito } a & \sum_{j \in N} x_{i j}=\sum_{j \in N} x_{j i}, \quad i \in P \\
& \sum_{j \in P} x_{s j}=\sum_{j \in P} x_{j t}=1 \\
& x_{i j}>0 \Rightarrow t_{i}+t_{i j} \leq t_{j}, \quad(i, j) \in I \\
& a_{i} \leq t_{i} \leq b_{i}, \quad i \in P \\
& x_{i, j} \in\{0,1\}, \quad(i, j) \in A .
\end{aligned}
$$




\subsection{Resolução do Subproblema}

A rota com o menor custo satisfazendo as restrições de programação é determinada usando-se uma adaptação do algoritmo de Ford-Bellman para problemas com restrições de tempo. Para maiores detalhes do Algoritmo de Ford-Bellman veja o Apêndice $C$.

Para resolvermos o problema de caminho mínimo com janela de tempo, as rotas têm que ser comparadas em termos de seu tempo de chegada até os nós. Para tratarmos simultaneamente as variáveis de tempo $t_{i}$ e as variáveis de custo $\pi_{i}$ associadas a cada nó $i$, utilizaremos a etiqueta $\left(t_{i}, \pi_{i}\right)$ para rotular cada nó $i$.

Uma etiqueta $\left(t_{i}, \pi_{i}\right)$ é atribuída ao nó $i$ se, e somente se, existir uma possível rota de custo $\pi_{i}$, saindo da origem $s$ e e chegando ao nó $i$, no menor tempo $t_{i}$ possível. Considere uma etiqueta $\left(t_{i}, \pi_{i}\right)$ associada a rota $X_{s_{i}}$ qualquer, com um custo $\pi_{i}$ e o tempo $t_{i}$ de chegada ao nó $i$ dentro do intervalo de tempo $\left[a_{i}, b_{i}\right]$.

$O$ algoritmo de Ford-Bellman associa a cada nó, somente uma etiqueta, a qual é progressivamente melhorada. Quando acrescentamos ao problema as restrições de janela de tempo, não é mais possível conservar a melhor etiqueta em cada nó, porque o par $\left(t_{i}, \pi_{i}\right)$ não possui ordem total no $R^{2}$. Podemos então, conservar o conjunto de etiquetas $\left(t_{i}^{\alpha}, \pi_{i}^{\alpha}\right), \alpha \geq 1$. Este conjunto será evidentemente encontrado, porque a quantidade finita de nós determina um número finito de possíveis caminhos da origem $s$ ao nó $i$, e cada possível caminho produz uma etiqueta. Desta forma, obtemos para o nó $i$ um conjunto de etiquetas e o denotamos por $Q_{i}$.

Para cada nó $i$, construímos um conjunto de etiquetas $Q_{i}$ que fornece as melhores distâncias em diferentes instantes dentro da janela de tempo $\left[a_{i}, b_{i}\right]$. Definimos então a relação de ordem parcial < para o nó $i$ :

$$
\left(t_{i}, \pi_{i}\right)<\left(t_{i}^{2}, \pi_{i}^{2}\right) \Leftrightarrow\left(t_{i} \leq t_{i}^{2}\right) \text { e }\left(\pi_{i} \leq \pi_{i}^{2}\right)
$$

Uma etiqueta pertencente ao conjunto $Q_{i}$ é denominada etiqueta mínima se não existir um outra etiqueta inferior a ela seguindo esta relação de ordem. Assim, as etiquetas mínimas guardam o comprimento do caminho ótimo da origem $s$ ao destino $i$. Uma etiqueta mínima é uma etiqueta ótima $\left(t_{i}^{*}, \pi_{i}^{*}\right)$, se quando aplicarmos o princípio de otimalidade a esta etiqueta, ela continuar com o menor caminho satisfazendo às restrições de janela de tempo em relação as outras etiquetas do conjunto. O resultado desta aplicação permite reduzir o conjunto de etiquetas de cada nó tirando aquelas que não são mínimas 
e conservando o conjunto das etiquetas irredutível dentro do tempo de atendimento permitido. O conjunto irredutível de etiquetas associado ao nó $i$ é ordenado seguindo uma ordem de tempo estritamente crescente, observando que os comprimentos $\pi_{i}$ estão em ordem decrescente.

O conjunto irredutível de etiquetas associado ao nó $i$ contém $\alpha(i)$ pares de etiquetas: $\left(t_{i}^{\alpha}, \pi_{i}^{\alpha}\right), \alpha=1,2, \ldots, \alpha(i)$, com

$$
a_{i} \leq t_{i}^{1}<t_{i}^{2}<\cdots<t_{i}^{\alpha(i)} \leq b_{i} \text { e } \pi_{i}^{1}>\pi_{i}^{2}>\cdots>\pi_{i}^{\alpha(i)}
$$

Para a etiqueta $\alpha, \pi_{i}^{\alpha}$ é a distância mais curta da origem $s$ ao nó i dentro do intervalo de tempo:

$$
\begin{aligned}
& {\left[t_{i}^{\alpha}, t_{i}^{\alpha+1}\right] \text { se } \alpha=1,2, \ldots, \alpha(i)-1} \\
& {\left[t_{i}^{\alpha(i)}, b_{i}\right] \text { caso contrário. }}
\end{aligned}
$$

\subsection{Algoritmo do Caminho Mínimo com Janela de Tempo}

Descrevemos então o algoritmo de caminho mínimo para uma rede com as restrições de janela de tempo em cada nó, de modo similar ao algoritmo de Ford-Bellman

Este algoritmo armazena em cada nó o par de etiqueta $\left(t_{i}, \pi_{j}\right)$, ou seja, uma rota de custo mínimo chegando ao nó $i$, no tempo $t_{i}$ ou antes.

Seja $Q_{i}=\left(t_{i}^{\alpha}, \pi_{i}^{\alpha}\right), \alpha=1,2, \ldots, \alpha(i)$ um conjunto irredutível de etiquetas associadas ao nó $i$. Unindo o arco $(i, j)$ aos caminhos que saem da origem $s$ e chegam em $i$, obtemos o conjunto $Q_{j}$ associado ao nó $j$ através da seguinte aplicação:

$$
F_{i j}\left(Q_{i}\right)=\bigcup_{\alpha} f_{i j}\left\{\left(t_{i}^{\alpha}, \pi_{i}^{\alpha}\right)\right\}
$$

onde

$$
f_{i j}\left\{\left(t_{i}^{\alpha}, \pi_{i}^{\alpha}\right)\right\}=\left\{\begin{array}{l}
\left(\max \left[a_{j}, t_{i}^{\alpha}+t_{i j}\right], \pi_{i}^{\alpha}+c_{i j}\right), \text { se } t_{i}^{\alpha}+t_{i j} \leq b_{j} \\
\emptyset, \text { caso contrário }
\end{array}\right.
$$


A definição de $f_{i j}$ permite um tempo de espera no nó $j$ quando o tempo de chegada ao nó $j$ for menor que o limitante inferior da janela de tempo. Assim, o novo conjunto $F_{i j}\left(Q_{i}\right)$ não é necessariamente irredutível pois os valores do tempo $t_{j}^{\alpha}$ podem ser aplicados para os valores de $a_{j}$ para as principais etiquetas:

$$
t_{j}^{\alpha}=a_{j}, \forall \alpha \text { tal que } t_{i}^{\alpha}+t_{i j}<a_{j} .
$$

Definimos $\overline{F_{i j}\left(Q_{i}\right) \cup Q_{j}}$ como a redução de dois conjuntos conservando apenas as etiquetas mínimas. As etiquetas associadas a um nó estão localizadas dentro de uma lista com os tempos em ordem crescente.

O cálculo de $\overline{F_{i j}\left(\overline{Q_{i}}\right) \cup Q_{j}}$ nos nós $j$ é feito por uma atualização de sequência do conjunto $Q_{j}$ a partir do conjunto $\triangle_{i}$ sobre o conjunto $Q_{i}$, onde $\triangle_{i}$ é o conjunto que contém as etiquetas modificadas depois que o nó $i$ está dentro do conjunto $L$; essas são as possíveis modificações do nó $j$. A atualização ocorre progressivamente dentro do conjunto $\triangle_{i}$ e $Q_{i}$, e depois é comparada com o comprimento de caminho mínimo saindo da origem $s$ e chegando ao nó $j$ utilizando os mesmos tempos.

A seguir mostraremos os principais passos do algoritmo.

Passo 1: Inicialização

$$
\begin{aligned}
& Q_{0}=\left\{\left(t_{s}^{1}=a_{s}, \pi_{s}^{1}=0\right)\right\} \\
& Q_{i}=\left\{\left(t_{i}^{1}=a_{i}, \pi_{i}^{1}=\infty\right)\right\} \text { para } i \neq j \\
& L=\{s\}
\end{aligned}
$$

passo 2: Explorando $F(i)$ do nó $i$

Construa $F(i)$, conjunto de sucessores do nó $i$

Para $i \in L$, faça

- $\forall j \in F(i)$ tal que $F_{i j}\left(Q_{i}\right) \bigcup Q_{j}$, onde

$$
F_{i j}\left(Q_{i}\right)=\bigcup_{\alpha} f_{i j}\left\{\left(t_{i}^{\alpha}, \pi_{i}^{\alpha}\right)\right\}
$$

$\mathrm{e}$

$$
f_{i j}\left\{\left(t_{i}^{\alpha}, \pi_{i}^{\alpha}\right)\right\}=\left\{\left(\max \left[a_{j}, t_{i}^{\alpha}+t_{i j}\right], \pi_{i}^{\alpha}+c_{i j}\right)\right\}, \text { tal que } t_{i}^{\alpha}+t_{i j} \leq b_{j}
$$


- Verifique as etiquetas mínimas

$$
\overline{F_{i j}\left(Q_{i}\right) \cup Q_{j}} \neq Q_{j}
$$

- Atualize $Q_{j}=\overline{F_{i j}\left(Q_{i}\right) \cup Q_{j}}, L=L \cup\{j\}$

Passo 3: Redução do conjunto $L$

$$
L=L-\{i\}
$$

Se $L=\emptyset$ então PARE

Senão, retorne ao Passo 2.

\subsection{Resolução da Formulação por Partição de Con- juntos}

O Método Simplex é utilizado para resolver a relaxação do problema de Partição de Conjunto. Segundo Desrosiers et al. (1984), os resultados iniciais mostraram que o tempo computacional gasto para resolver o Método Simplex é bem maior que resolver o problema do caminho mínimo com janela de tempo. Para acelerar a convergência, em vez de gerar um rota, Desrosiers et al. (1984) geraram várias rotas em cada iteração do Método Simplex. Esta modificação explora a idéia de que é importante incluir no conjunto das rotas geradas, aquelas rotas com grande probabilidade de serem ótimas. Esta alteração reduz o esforço computacional do método simplex enquanto aumenta o uso problema do caminho mínimo com janela de tempo, o qual é relativamente barato.

A. solução da relaxação do problema de partição de conjunto pode não ser inteira. Neste caso é preciso realizar ramificacooes para obter a solução inteira. Suponha que a variável fracionária seja $y_{r}$. Então teremos duas ramificações

$$
y_{r}=1 \text { e } y_{r}=0 .
$$

A ramificação $y_{r}=0$ pode destruir a estrutura do subproblema. Isto também aconteceu com o Problema de Designação Generalizada, visto no Capítulo 03. Tomando $y_{r}=0$, significa que a rota $\delta_{\tau}$ é excluída. Entretanto é possível que a próxima vez que o Problema de Caminho com Janela de Tempo for resolvido, tenhamos novamente a rota $\delta_{r}$ 
como solução. Neste caso, é necessário determinar a segunda melhor rota. Para suprir essa dificuldade, a ramificação é executada na variável $x_{i j}$ que faz parte da rota.

Se $y_{r}=1$, significa que a rota $\delta_{r}$ será utilizada e que as novas rotas que serão geradas não precisam incluir os nós percorridos durante a rota $\delta_{r}$. Neste caso, os nós da rota $\delta_{r}$ são removidos do subproblema.

Se $y_{r}=0$, significa que a rota $\delta_{r}$ não será utilizada em nenhum momento. Neste caso precisamos tomar o cuidado para que a rota $\delta_{r}$ não seja gerada no subproblema. Isto pode ser evitado de várias maneiras. Suponha que a rota $\delta_{r}$ passe pelos nós $1,2 \mathrm{e}$ 3. Para evitar que a rota se repita, podemos remover o arco $(2,3)$ ou o arco $(1,2)$ do subproblema. Podemos também retirar algum nó da rota $\delta_{\tau}$ no subproblema. O número de possibilidades de evitar que se repita a rota cresce exponencialmente de acordo com o tamanho da rota.

Uma vez realizada a ramificação, novas colunas são geradas para encontrar a solução ótima. 


\section{Capítulo 5}

\section{Experiências Computacionais}

\subsection{Introdução}

Neste capítulo será apresentado as experiências computacionais. O modelo utilizado é o proposto por Desrosiers et al. (1984) para o problema de Roteamento de Veículos com Janela de Tempo.

\subsection{Descrição do Teste}

Como visto no Capítulo 4, o Problema de Roteamento com Janela de Tempo pode ser formulado como um problema de Partição de Conjunto da forma

$$
\begin{array}{lll}
\text { Minimizar } & \sum_{r \in R} c_{r} y_{\mathrm{r}} & \\
\text { Sujeito a } & \sum_{r \in R} \delta_{r i} y_{\mathrm{r}}=1, & i \in P \\
& y_{\mathrm{r}} \in\{0,1\}, \quad r \in R .
\end{array}
$$

onde $R$ é o conjunto de rotas, $P$ o conjunto de clientes, $\delta_{r i}$ a constante binária que toma valor igual a 1 se a rota $r \in R$ inclui a viagem $i \in P$ e 0 caso contrário, $c_{r}$ o custo da rota $r \in R$ e $y_{\mathrm{r}}$ a variável binária que toma valor igual a 1 se a rota $r \in R$ é utilizada e 0 caso contrário.

A relaxação do problema (5.1) será resolvida utilizando o Método Simplex Revisado com Geração de Colunas. Rotas adicionais adicionais serão geradas resolvendo o problema do Caminho Mínimo com Janela de Tempo. 
Para os experimentos computacionais serão utilizados 4 problemas testes. Os problemas testes são versões menores dos problemas desenvolvido por Solomon (1987). Solomon (1987) desenvolveu um conjunto de problemas com 101 nós (100 clientes e 1 depósito) e separados de acordo com a distribuição dos dados geográficos: nós uniformemente distribuídos $(R 1)$, nós agrupados em blocos $(C 1)$ e uma mistura de $R 1$ e $C 1$ ( $R C 1)$. As distância e o tempo entre os nós são determinadas pela distância Euclidiana e o truncamento da mesma, respectivamente. Os problemas testes têm 26 nós ( 25 clientes e 1 depósito ). Dentre os 4 problemas, 2 são da categoria R1 e 2 da categoria C1. Não é considerado a demanda em cada nó. A razão por utilizar uma versão menor dos problemas propostos por Solomon (1987) será descrita posteriormente.

O algoritmo foi programado utilizando a linguagem $C$ e os testes foram executados num computador Pentium II, 266 com 64 MB de memória RAM.

A partição básica inicial para o problema (5.1) é obtida tomando as rotas que passam precisamente por um único nó. Neste caso, a partição básica é a matriz identidade. Para gerar novas rotas são utilizados dois procedimentos:

Regra 1: selecionamos a rota com o menor custo relativo;

Regra 2: selecionamos a rota com o menor custo relativo e os nós percorrido pela rota são removidos para que rotas disjuntas sejam geradas.

Segundo Desrosiers et al, a construção de rotas disjuntas favorece o aparecimento de soluções inteiras.

$\mathrm{Na}$ Tabela 5.1 temos os resultados obtidos.

\begin{tabular}{|c|c|c|c|c|c|c|}
\hline & & Regra 1 & & & Regra 2 & \\
\hline Problema & Solução & Solução Ótima & Iterações & Solução & Solução Ótima & Iterações \\
\hline R125-01 & 561,75 & Não & 116 & 614,37 & Sim & 19 \\
\hline R125-02 & 612,85 & Sim & 130 & 667,40 & Sin & 12 \\
\hline C125-01 & 220,88 & Não & 1600 & 632,33 & Não & 5 \\
\hline C125-02 & 372,34 & Sim & 339 & 844,17 & Não & 7 \\
\hline
\end{tabular}

Tabela 5.1: resultados obtidos

Para os problemas R125-01 e R125-02, a utilização da Regra 2 forneceu uma solução 
ótima inteira e com um número baixo de iterações. Para os problemas C125-01 e C125-02, a Regra 2 não forneceu um solução boa para o problema relaxado. A solução ótima inteira foi obtida utilizando a Regra 1 no problema C125-02. O problema C125-02 é formado por 4 blocos de clientes e foram gerados exatamente 4 rotas, sendo cada rota percorrendo cada bloco.

Uma grande dificuldade encontrada na resolução do subproblema do Caminho Mínimo com Janela de Tempo foi a geração de rotas com ciclos. Pare este subproblema, temos que os custos podem ser negativos e neste caso podemos ter rotas com ciclos. Esses ciclos são finitos, pois a janela de tempo impede que os ciclos se repitam infinitamente. Por exemplo, se uma determinada rota $\delta_{t}$ passar 3 vezes no nó $i$, teremos $\delta_{t i}=3$. As rotas com ciclos não farão parte da solução ótima inteira, pois as variáveis associadas a tais rotas nunca poderão tomar valor igual a 1 (devido à formulação por Partição de Conjuntos do problema).

No algoritmo do Caminho Mínimo com Janela de Tempo, em cada nó é armazenado numa lista todas as possíveis etiquetas. A quantidade de etiqueta em cada nó é grande e pode variar a cada iteração do Método Simplex. Na implementação do algoritmo, o tamanho da lista é um valor fixo para cada nó. Se o número de nós é muito grande, ao fixar o tamanho da lista, a memória computacional fica bastante limitada. Isto foi o principal motivo para utilizar versões menores dos problemas desenvolvido por Solomon (1987). Uma alternativa para esse problema seria utilizar o conceito de ponteiro nas implementações das listas, pois neste caso, não será necessário fixar tamanho da lista. 


\section{Capítulo 6}

\section{Conclus es e Propostas para}

\section{Futuro}

Este trabalho abordou o estudo da técnica de geração de colunas em diversos problemas de otimização. Inicialmente, a técnica de geração de colunas foi utilizada para resolver problemas de Otimização Linear de grande porte e atualmente é utilizada para resolver problemas de Otimização Inteira.

Em problemas de Otimização Linear de grande porte, a dificuldade de resolver tais problemas pelo Método Simplex existe em decorrência do número excessivamente grande de variáveis. A geração de colunas tem a vantagem de trabalhar com um número limitado de colunas e novas colunas serão geradas, quando necessário, a partir da resolução de um subproblema. Este subproblema tem a vantagem de possuir parte da característica combinatória do problema original.

A resolução de problemas de Otimização Inteira requer formulações cuja relaxações forneçam uma aproximação boa para o envoltório convexo das soluções factíveis. A relaxação do Problema Reformulado (aplicação de Dantzig e Wolfe no Problema Original) fornece um limitante melhor que o limitante fornecido pela relaxação do Problema Original. Em comparação com a Relaxação Lagrangiana, os limitantes fornecidos são idênticos. De acordo com Barhart et al. (1998), os resultados obtidos pela relaxação do Problema Reformulado superam a Relaxaçāo Lagrangiana. Isto acontece em decorrência dos códigos simplex modernos.

A utilização do branch and bound na relaxação do Problema Original pode realizar ramificações "pobres", devido à repetição de soluções simétricas (idênticas, do ponto de 
vista do modelo) na árvore de enumeração. Isto pode ser evitado utilizando-se a relaxação do Problema Reformulado.

As ramificações realizadas na relaxação do Problema Original devem ser bem elaboradas para que não alterem a estrutura do subproblema. Ou seja, devemos evitar que as colunas infactíveis devido às restrições de ramificação não sejam geradas, e que, ao acrescentarmos as restrições de ramificação o subproblema não se torne mais difícil de ser resolvido. Em problemas de Particionamento de Conjunto, as restrições de ramificação correspondem a remover variáveis do subproblema, de tal forma que as colunas infactíveis não serão geradas. Segundo Barhart et al. (1998), geralmente não é possível remover as variáveis do subproblema para eliminar as colunas infactíveis no Problema Reformulado Geral. Neste caso as restrições de ramificação são acrescentadas ao Problema Reformulado Geral, tornando a resolução do subproblema mais difícil.

A principal proposta para o futuro será resolver o Problema de Roteamento com Janela de Tempo utilizando o algoritmo Branch and Price. Uma grande dificuldade encontrada no Problema de Roteamento com Janela de Tempo foi o problema da ciclagem na resolução do subproblema do Caminho Mínimo com Janela de Tempo. Ao considerar a ciclagem, uma alternativa seria de formular o modelo como um problema de Cobertura de Conjunto. Desrochers et al. (1992) apresentam um algoritmo para o Problema de Caminho Mínimo com Janela de Tempo que evita ciclos o qual poderá ser utilizado para resolver o subproblema. Para melhorar a eficiência do subproblema é necessário implementar o algoritmo do problema do Caminho Mínimo com Janela de Tempo utilizando ponteiros.

O desenvolvimento de estratégias de ramificação para o algoritmo Branch and Price é uma área de constante pesquisa. Vanderbeck (2000) propõe várias estratégias de ramificação, as quais poderão ser utilizando em vários problemas da literatura.

$\mathrm{Na}$ literatura encontram-se poucos trabalhos que relacionam o algoritmo de geração de colunas e geração de planos de corte (Branch and Price and Cut). O estudo dessa técnica de resolução pode contribuir para desenvolvimento de novos algoritmos na área de Otimização Inteira. 


\section{Apêndice A}

\section{O Método Simplex}

\section{A.1 Revisão do Método Simplex}

Considere o Problema Primal de Otimização Linear:

$$
\begin{array}{ll}
\text { Minimizar } & f(x)=c^{T} x \\
\text { Sujeito a } & A x=b \\
& x \geq 0
\end{array}
$$

onde $A$ é uma matriz $m \times n \operatorname{com} P o s t o(A)=m$ e $b$ é um vetor $m$-dimensional.

Suponha que o $\operatorname{Posto}(A, b)=\operatorname{Posto}(A)$. A solução geral do sistema pode ser descrita da seguinte maneira. Considere uma partição das colunas da matriz $A$ :

$$
A=(B, N)
$$

onde $B$ é uma matriz $m \times n$ não-singular formada por $m$ colunas da matriz $A$ e $N$ é uma matriz $m \times(m-n)$. A mesma partição é feita no vetor das variáveis $x=\left(x_{B}, x_{N}\right)^{T}$, onde $x_{B}$ é chamado vetor das variáveis básicas e $x_{N}$ vetor das variáveis não básicas.

Assim, podemos escrever o sistema $A x=b$ da seguinte forma:

$$
\begin{aligned}
& A x=b \\
& B x_{B}+N x_{N}=b \\
& x_{B}=B^{-1} b-B^{-1} N x_{N}
\end{aligned}
$$


Definição 1: A solução particular de $x$ obtida por: $x_{B}=B^{-1} b$ e $x_{N}=0$, é chamada solução básica. Se $x_{B}=B^{-1} b \geq 0$, então a solução básica é primal-factível e dizemos que a partição básica também é primal-factível.

Considere também a partiçāo básica nos coeficientes da função objetivo $c^{T}$ :

$$
c^{T}=\left(c_{B}, c_{N}\right)^{T}
$$

Definição 2: Chamamos o vetor $\pi \in R^{m}$, dado por

$$
\pi^{T}=c_{B}^{T} B^{-1}
$$

por vetor multiplicador simplex. Se

$$
c_{j}-\pi^{T} a_{j} \geq 0, j=1, \ldots, n
$$

então $\pi$ é uma solução básica dual-factível. Observe que, pela definição de $\pi, \pi^{T} a_{j}=c_{j}$ para todo $a_{j} \in B$. Neste caso dizemos que a partição básica é dual-factível.

Teorema 1 1. Quando uma partição básica for primal e dual factível, então as soluções básicas associadas resolvem o problema primal e dual, respectivamente, e dizemos que a partição é ótima.

A solução geral do sistema (A.2) permite escrever também a função objetivo em termos das variáveis não básicas, ou seja

$$
\begin{aligned}
f(x) & =c^{T} x \\
& =c_{B} x_{B}+c_{N} x_{N} \\
& =c_{B}\left(B^{-1}-B^{-1} N x_{N}\right)+c_{N} x_{N} \\
& =f\left(x^{0}\right)+\sum_{j \in N}\left(c_{j}-\pi^{T} a_{j}\right) x_{j},
\end{aligned}
$$

o que mostra como a função objetivo, restrita ao sistema $A x=b$, se altera quando fazemos perturbações na solução factível.

Definição 3: Chamamos de estratégia simplex a seguinte perturbação da solução básica inicial:

- Escolha $k \in N$, tal que: $c_{k}-\pi^{T} a_{k}<0$; 
- Faça $x_{k}=\varepsilon \geq 0$ e $x_{j}=0, \vee j \in N-k$.

A estratégia simplex produz uma nova solução dada por :

$$
\left\{\begin{array}{l}
x_{B}=x_{B}^{0}+\epsilon y \\
x_{N}=\epsilon e_{k}
\end{array}\right.
$$

onde $y=-B^{-1} a_{k}$ e $e_{k}=(0, \ldots, 1, \ldots 0)^{T} \in R^{n-m}$, com 1 na $k$-ésima componente. O valor da função objetivo será dada por:

$$
f(x)=f\left(x^{0}\right)+\left(c_{k}-\pi^{T} a_{k}\right) \epsilon
$$

cuja expressão $c_{k}-\pi^{T} a_{k}$ é chamada de custo relativo.

Note que a direção $d \in R^{n}$, dada por $d=\left(d_{B}, d_{n}\right)^{T}=\left(y, e_{k}\right)^{T}$, define uma perturbação da soluçāo básica e é chamada direção simplex. Se a solução for não degenerada, ou seja $x_{B}^{0}>0$, de (A.2) e (A.1) segue que $d$ é uma direção factível. Note ainda, que o produto escalar da direção $d$ com o gradiente da função objetivo é $c^{T} d=c+k-\pi^{T} a_{k}<0$ e se o índice $k$ for escolhido tal que $c^{T} d<0$, então a partir de (A.1) temos que a direção é de descida.

De (A.1), podemos determinar o maior valor do passo $\epsilon$, impondo $x_{B} \geq 0$ :

$$
\epsilon^{0}=\frac{x_{B_{l}}^{0}}{y_{l}}=\operatorname{Minimo}\left\{\frac{x_{B_{i}}^{0}}{y_{i}} \mid y_{i}<0, i=0, \ldots, m\right\}
$$

onde $x_{B_{i}}$ é a i-ésima componente de $x_{B}^{0}$.

Para esta escolha de $\epsilon^{0}$, a l-ésima componente de $x_{B}$ se anula $\left(x_{B_{r}}=x_{B_{r}}^{0}-\frac{x_{B_{r}}^{0}}{y_{r}} y_{r}=0\right)$, enquanto apenas uma variável de $x_{N}$ torna-se positiva $\left(x_{N}=\epsilon\right)$. Isto sugere uma nova partição básica, conforme o seguinte teorema.

Teorema 1 2. Considere a nova partição básica $A=\left(B^{\prime}, N^{\prime}\right)$ onde a k-ésima coluna de $B^{\prime}$ é dada por $a_{k} \in N$. Temos que a nova partição básica factível $e$ a sua solução associada é dada por: $x^{1}=x^{0}+\epsilon d$.

De (A.1), podemos escolher qualquer $k$ tal que $c_{k}-\pi^{T} a_{k}<0$. Um dos critérios mais utilizados para escolher o índice $k$, é o critério da Máxima Descida de Dantzig. O critério de Dantzig escolhe o índice $k$ tal que

$$
c_{k}-\pi^{T} a_{k}=\text { Mínimo }\left\{c_{j}-\pi^{T} a_{j}, j=1, \ldots, n\right\}
$$


Se $c_{k}-\pi^{T} a_{k} \geq 0$, então pelo Teorema 1 , a corrente solução básica factível é uma solução ótima.

Desta forma, enquanto a solução básica dual for infactível, podemos repetir o procedimento descrito acima. Assim, tal procedimento descreve o Método Simplex.

A seguir é dado o algoritmo Primal Simplex.

\section{A.2 Algoritmo Primal Simplex}

Fase I: Encontre uma partição o básica primal-factível: $A=(B, N)$

(Se necessário, utilizar o método das variáveis artificiais)

Faça PARE $=$ FALSO e $I T=0$

Fase II: Enquanto NĀO PARE, faça:

Passo 1: Determine a solução o básica primal factível: $x_{B}=B^{-1} b$

Passo 2: Teste de Otimalidade

- Determine a solução o básica dual: $\pi^{T}=c_{B}^{T} B^{-1}$

- Determine o menor custo relativo

$$
c_{k}-\pi^{T} a_{k}=\text { Mínimo }\left\{c_{j}-\pi^{T} a_{j}: j=1,2, \ldots, n\right\}
$$

- Se $c_{k}-\pi^{T} a_{k} \geq 0$, então a solução na iteração o IT é ótima PARE $=$ VERDADE

Passo 3: Determine a direção o Simplex: $y=-B^{-1} a_{k}$

Passo 4: Determine o valor

$$
\epsilon^{0}=\frac{x_{B_{l}}^{0}}{y_{l}}=\text { mínimo }\left\{\frac{x_{B_{i}}^{0}}{y_{i}} \mid y_{i}<0, i=1, \ldots, m\right\}
$$

Se $\mathrm{y} \geq 0$, então o problema não tem solução ótima finita.

PARE $=$ VERDADE

Passo 5: Atualize a partição o básica: $a_{B_{l}} \leftrightarrow a_{k}, I T=I T+1$. 


\section{Apêndice B}

\section{Decomposição de Dantzig e Wolfe}

Dantzig e Wolfe (1960) desenvolveram uma técnica, chamada princípio da decomposição de Dantzig e Wolfe, para resolver problemas de Otimização linear de grande porte e problemas de Otimização Linear que contenham restrições com estruturas especiais. As restrições podem ser divididas em dois conjuntos: restrições gerais (ou restrições complicadas) e restrições com estruturas especiais. A estratégia da decomposição é operar nos dois conjuntos de restrições. O problema linear sobre as restrições gerais é chamado Problema Mestre e o problema linear sobre as restrições especiais é chamado de subproblema. Para descrever o método, vamos considerar o problema de Programação Linear escrito na forma:

$$
\begin{array}{ll}
\text { Minimizar } & c^{T} x \\
\text { Sujeito a } & A x \leq b \\
& D x \leq d \\
& x \geq 0 .
\end{array}
$$

Vamos supor que a matriz do problema original (B.1) tenha a estrutura bloco-angular:

$$
B=\left[\begin{array}{llll}
A_{1} & A_{2} & \cdots & A_{N} \\
D_{1} & & & \\
& D_{2} & & \\
& & \ddots & \\
& & & D_{3}
\end{array}\right]
$$


Particionando os vetores $x, c$ e $d$ de acordo com a estrutura da matriz $B$, o problema (B.1) pode ser reescrito como

$$
\begin{array}{ll}
\text { Minimizar } & \sum_{i=1}^{N} c_{i} x_{i} \\
\text { Sujeito a } & \sum_{i=1}^{N} A_{i} x_{i} \leq b \\
& D_{i} x_{i} \leq d_{i}, \quad i=1, \cdots, N \\
& x_{i} \geq 0, \quad i=1, \cdots, N .
\end{array}
$$

O problema (B.2) pode ser visto como um problema de minimizar o custo total de $N$ diferentes subproblemas lineares, que são independentes, exceto pela primeira restrição. Cada i-ésimo sub́problema é da forma

$$
\begin{array}{ll}
\text { Minimizar } & c_{i} x_{i} \\
\text { Sujeito a } & D_{i} x_{i} \leq d \\
& x_{i} \geq 0
\end{array}
$$

$\operatorname{com} i=1, \cdots, N$.

Considere o conjunto $X_{i}=\left\{x_{i}: D_{i} x_{i} \leq d\right\}$. Vamos supor por simplicidade que $o$ conjunto $X_{i}$ seja limitado. Seja $\left\{x_{1}^{i}, x_{2}^{i}, \cdots, x_{K_{i}}^{i}\right\}$ o conjunto de pontos extremos de $X_{i}$. Então qualquer ponto de $X_{i}$ pode ser representado como uma combinação convexa de seus pontos extremos, ou seja, cada $x_{i} \in X_{i}$ é escrito como

$$
\begin{aligned}
& x_{i}=\sum_{j=1}^{K_{i}} \lambda_{j}^{i} x_{j}^{i} \\
& \sum_{\substack{j=1 \\
K_{i}}}^{\lambda_{j}^{i} \geq 0,} \lambda_{j}^{i}=1, \quad i=1, \cdots, N \\
& \quad i=1, \cdots, N, \quad j=1, \cdots, K_{i} .
\end{aligned}
$$

Correspondendo a cada ponto extremo $x_{j}^{i}$, defina $p_{j}^{i}=c_{j} x_{j}^{i}$ e $q_{j}^{i}=A_{i} x_{j}^{i}$. Temos que $p_{j}^{i}$ é o custo de cada ponto extremo $x_{j}^{i}$ e $q_{j}^{i}$ é o vetor equivalente à restrição de acoplagem.

Então o problema original (B.1) é equivalente ao problema 


$$
\begin{array}{ll}
\text { Minimizar } & \sum_{i=1}^{N} \sum_{j=1}^{K_{i}} p_{j}^{i} \lambda_{j}^{i} \\
\text { Sujeito } a & \sum_{i=1}^{N} \sum_{j=1}^{K_{i}} q_{j}^{i} \lambda_{j}^{i} \leq b \\
& \sum_{j=1}^{K_{i}} \lambda_{j}^{i}=1, \quad i=1, \cdots, N \\
& \lambda_{j}^{i} \geq 0, \quad i=1, \cdots, N, \quad j=1, \cdots, K_{i} .
\end{array}
$$

O problema (B.4) é chamado de Problema Mestre.

Como as variáveis do Problema Mestre (B.4) são

$$
\lambda=\left\{\lambda_{1}^{1}, \ldots, \lambda_{K_{1}}^{1}, \lambda_{1}^{2}, \ldots, \lambda_{K_{2}}^{2}, \lambda_{1}^{n}, \ldots, \lambda_{K_{n}}^{n}\right\}
$$

o problema pode ser escrito mais compactamente por

$$
\begin{array}{ll}
\text { Minimizar } & p^{T} \lambda \\
\text { Sujeito a } & Q \lambda \leq g \\
& \lambda \geq 0
\end{array}
$$

com $g^{T}=\left(b_{0}^{T}, 1, \ldots, 1\right), p=\left\{p_{1}^{1}, \ldots, p_{K_{1}}^{1}, p_{1}^{2}, \ldots, p_{K_{2}}^{2}, p_{1}^{n}, \ldots, p_{K_{n}}^{n}\right\}$ e cada coluna de $Q$ associada a $\lambda_{i}^{j}$ sendo da forma $\left(q_{j}^{i} e_{i}\right)^{T}$.

Para aplicar o Método Simplex ao Problema Mestre (B.4), vamos supor que é dada a partição $Q=(B, N)$ e o vetor multiplicador simplex $\pi=p_{B}^{T} B^{-1}$. O custo relativo de cada coluna é

$$
r_{i j}=p_{j}^{i}-\pi^{T}\left[\frac{q_{j}^{i}}{e_{i}}\right]
$$

Para determinar a coluna que entrará na base, precisamos determinar o menor dos custos relativos. A procura do elemento com o menor custo relativo é feita sobre as colunas não básicas, podendo ser estendida às colunas básicas, pois para as variáveis básicas sempre teremos

$$
r_{i j}=p_{j}^{i}-\pi^{T}\left[\frac{q_{j}^{i}}{e_{i}}\right]=0
$$

Seja $r^{*}$ o menor dos custos relativos, isto é, 


$$
r^{*}=\text { Minimo }_{1 \leq i \leq N}\left\{r_{i}^{*}=\text { Minimo }_{1 \leq j \leq K_{i}}\left(p_{j}^{i}-\pi^{T}\left[\frac{q_{j}^{i}}{e_{i}}\right]\right)\right\}
$$

Da definição de $p_{j}^{i}$ e $q_{j}^{i}$ temos:

$$
\begin{aligned}
r^{*} & =\text { Mínimo }\left\{c_{i} x_{j}^{i}-\pi_{0}^{T} L_{i} x_{j}^{i}-\pi_{m+1}\right\} \\
& =\text { Mínimo }\left\{\left(c_{i}-\pi_{0}^{T} L_{i}\right) x_{j}^{i}-\pi_{m+1}\right\}
\end{aligned}
$$

onde o vetor $\pi_{0}^{T}$ é constituído pelos primeiros $m$ elementos de $\pi$ e $m$ é o número de linhas de $L_{i}$.

A enumeração de todos os pontos extremos é uma tarefa difícil, pois em problemas de grande porte, o número de pontos extremos pode ser muito grande. Desse modo, o problema de determinar o menor dos custos relativos pode ser determinado pelo $i$-ésimo subproblema

$$
\begin{array}{ll}
\text { Minimizar } & \left(c_{i}-\pi_{0}^{T} L_{i}\right) x_{j}^{i} \\
\text { Sujeito } a & D_{i} x_{i} \leq d_{i} \\
& x_{i} \geq 0
\end{array}
$$

A solução deste subproblema fornecerá um vértice de $S_{i}$, a partir do qual uma coluna do Problema Mestre será gerada. Se o custo relativo for negativo, a variável associada a coluna entra na base e uma nova iteração do Método Simplex é executada. 


\section{Apêndice C}

\section{Algoritmo de Ford-Bellman}

O algoritmo de Ford-Bellman é um procedimento para determinar a rota com menor custo ou menor distância entre duas cidades (ou nós). O algoritmo de Ford-Bellman, determina para cada nó uma etiqueta $\pi_{i}$ que representa o custo de ir da origem $s$ paro o nó $i$ utilizando a rota de custo mínimo. As etiquetas são melhoradas por programação dinâmica até que os valores ótimos sejam obtidos;

Seja $F(i)=\{j:(i, j) \in A, j \in N\}$ o conjunto de sucessores do nó $i$ e $L$ o conjunto de nós que ainda não foram explorados. A árvore de caminho mínimo saindo da origem $s$ para todos os outros nós da rede é obtida associando-se a cada nó uma etiqueta que é progressivamente melhorada. O método de colocar as etiquetas consiste na escolha de um nó $i$ pertencente ao conjunto $L$, em seguida retira-se o nó $i$ do conjunto $L$ e tenta-se melhorar todos os sucessores do nó $i$. Logo o novo nó melhorado é inserido no conjunto $L$. Assim, quando o conjunto $L$ for esgotado, a etiqueta $\pi_{i}$ associada ao nó $i$ representará o caminho de comprimento mínimo saindo da origem $s$ e terminando no nó $i$.

A seguir temos os principais passos do algoritmo de Ford-Bellman.

Passo 0: Inicialização

Custo na origem $s: \pi_{s}=0$

Custo nos demais nós: $\pi_{i}=\infty$

$L=\{s\}$

Passo 1: Exploração de $F(i)$

Construa $F(i)$, conjunto de sucessores do nó $i$ 
Para $i \in L$, faça:

- $\forall j \in F(i)$, onde $F(i)=\{j:(i, j) \in A, j \in N\}$ tal que $\pi_{j}>\pi_{i}+\pi_{i j}$ atualize $\pi_{j}=\pi_{i}+c_{i j}$

- $L=L \cup\{j\}$

Passo 2: Redução do conjunto $L$

$L=L-\{i\}$

Se $L=\emptyset$ então PARE

Senão, retorne ao Passo 1 


\section{Referências Bibliográficas}

Assad, A.A. Modeling and Implemention Issues in Vehicle Routing. Vehicle Routing: Methods and Studies, North Holland, Amsterdam, 7-46, 1988.

Barnhart, C.; Johnson E.L.; Nenhauser, G.L.; Savelsbergh M.W.P.; Vance, P.H. Branchand-Price: Column Generation for Solving Huge Integer Programs. Operations Research, 46, 3, 316-329, 1998.

Barnhart, C.; J ohnson E.L.; Nenhauser, G.L.; Vance, P.H. Solving Binary Cutting Stock Problems by Column Generation and Branch and Bound. Computacional Optimization and Applications, 3, 111-130, 1994.

Bramel, J.; Levi, D.S. On the Effectiveness of Set Covering Formulations for the Vehicle Routing Problem With Time Windows. Operations Research, 45, 295-301, 1997.

Bodin, L.D.; Golden, B.; Assad, A.; Ball, M. Routing and Scheduling of Vehicles and Crews: The State of the Art. Computers and Operations Research, 10, 2,1983.

Brito, J.A.M.; Loiseu, I.; Maculan, N.; Passini, M.M. Column-Generation in Integer Linear Programming. Working Paper, 1-15, 2000.

Carvalho, J. M. V. Exact Solution of Cutting Stock Problems Using Column Generation and Branch and Bound. International Transactions in Operations Research, 40, 2, 342$353,1998$.

Cattrysse, D.; Kuik, R.; Salomon, M.; Wassenhove, L.N.V. A Dual Ascent and Column 
Generation Heuristic for the Discrete Lotsizing and Scheduling Problem with Setup Times. Management Science, 39, 4, 477-486, 1993.

Dantzig, G.B.; Fulkerson, D.R. Minimizing the Number of Tankers to Meet a Fixed Schedule. Naval Research Logistics Quart., 1, 217-222, 1954.

Dantzig, G. B.; Wolfe, P. Decomposition Principle for Linear Programming. Operations Research, 8, 101-111, 1960.

Desrochers, M.; Desrosiers, J.; Soumis, F. Routing with Time Windows by Column Generation, Networks, 10, 545-565, 1984.

Desrochers, M.; Desrosiers, J.; Solomon, M. N. A New Optimization Algorithm for the Vehicle Routing Problem With Time Windows. Operations Research, 40, 2, 343-353, 1992.

Desrosiers, J.; Dumas, Y.; Solomon, M. N.; Soumis, F. Time Constrained Routing and Scheduling. Network Routing - Handbook in Operations Research and Management Science, $8,35-139,1995$.

Desrosiers, J. Gamache, M.; Marquis, G.; Soumis, F. A Column Generation Approach for Large-Scale Aircrew Rostering Problems. Operations Research, 47, 2, 247-263, 1999.

Foster, B.A.; Ryan, D.M. An Integer Programming Approach to Scheduling. Computer Scheduling of Public Transport Urban Passenger Vehicle and Crew Scheduling. A. Wren, North-Holland, Amsterdam, 269-280, 1981.

Gilmore, P.C.; Gomory, R.E. A Linear Programming Approach to the Cutting Stock Problem. Operations Research, 9, 849-859, 1961.

Gilmore, P.C.; Gomory, R.E. A Linear Programming Approach to the Cutting Stock Problem - Part II. Operations Research, 11, 863-888, 1963. 
Lobel, A. Vehicle Scheduling in Public Transit and Lagrangean Pricing. Management Science, 44, 12, 1637-1649, 1998.

Johnson, E.L.; Nemhauser, G.L.; Savelsbergh, W. P. Progress in Linear ProgrammingBased Algorithms for Integer Programming: An Exposition. INFORMS Journal on Computing, 12, 1, 2-23, 2000.

Johnson, E.L. Modeling and Strong Linear Programs for Mixed Integer Programmig. Algorithms and Model Formulations in Mathematical Programming. S. W. Wallace (ed.). NATO ASI Series, 51, 1-41, 1989.

Mehrotra, A.; Trick M.A. A Column Generation Approach for Graph Coloring. INFORMS Journal on Computing, 8, 4, 1996.

Mehrotra, A.; Trick M.A. Cliques and Clustering: A Combinatorial Approach. Operations Research Letters, 22, 1-12, 1998.

Nemhauser, G. L.; Wolsey, L. A. Integer and Combinatorial Optimization, Wiley, New York, NY, 1988.

Ribeiro, C.C.; Soumis, F. A Column Generation Approach to the Multiple-Depot Vehicle Scheduling Problem. Operations Research, 42, 41-52, 1994.

Savelsbergh, M. A Branch-and-Price Algorithm for the Generalized Assigment Problem. Operations Research, 46̈, 6, 831-841, 1997.

Solomon, M.M. On the Worst-Case Performance of Some Heuristics for the Vehicle and Scheduling With Time Window Constraints. Networks, 16, 161-174, 1986.

Solomon, M.M. Algorithm for the Vehicle Routing and Scheduling Problem With Time Windows Constraints. Operations Research, 35, 254-265, 1987.

Ssierksma G., Tijssen. G. A. Routing Helicopters for Crew Exchanges on Off - Shore 
Locations. Annals of Operations, 76, 261-286, 1998.

Vance, P. Branch and Price Algorithms for the One-Dimensional Cutting Stock Problem. Computacional Optimization and Applications, 9, 211-228, 1998.

Van Den Akker, J.M.; Hurkens, C.A.J.; Savelsbergh M.W.P. Time-Indexed Formulations for Machine Scheduling Problems: Column Generation. INFORMS Journal on Computing, 12, 2, 111-124, 2000.

Vanderbeck, F. Computacional Study of a Column Generation Algorithm for Bin Packing and Stocking Problems. Mathematical Programming, 86, 565-594, 1999.

Vanderbeck, F. On Dantzig-Wolfe Decomposition in Integer Programming and Ways to Perform Branching in a Branch and Price Algorithm. Operations Research, 48, 1, 111-128, 2000.

Vanderbeck, F. Wolsey, L.A. An Exact Algorithm for IP Column Generation. Operations Research Letters, 19, 151-159, 1996. 